LBL $-\mathbf{2 8 8 1 8}$

DE90 016632

\title{
Sensitivity Studies on Parameters Affecting Gas Release from an Underground Rock Cavern
}

\author{
Erika Schlueter and Karsten Pruess \\ Earth Sciences Division \\ Lawrence Berkeley Laboratory \\ University of California \\ Berkeley, California 94720
}

January 1990)

This work was jointly supported by the U.S. Department of Energy under Contract No. DE-AC0376 SF00098 and by the Nationale Genossenschaft fü die Lagerung radioaktiver Abfaelle (NAGRA), Baden, Swizerland. 


\section{Contents}

1 INTRODUCTION 1

2 MODEL DOMAIN AND COMPUTATIONAL GRID 2

3 SYSTEM BEHAVIOR FOR THE REFERENCE CASE

4 SENSITIVITY STUDIES $\quad 11$

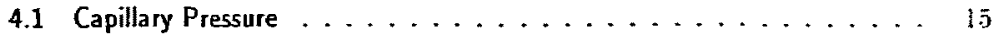

4.2 Relative Permeability . . . . . . . . . . . . . . . . 15

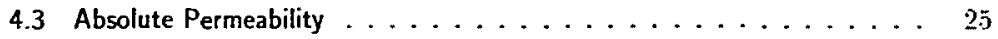

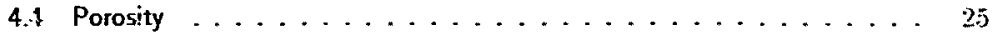

5 SUMMARY OF MULTPHASE EFFECTS 25

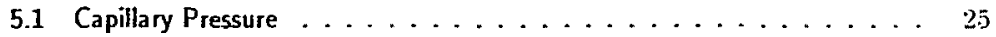

5.2 Relative Permeability . . . . . . . . . . . . . . . 33

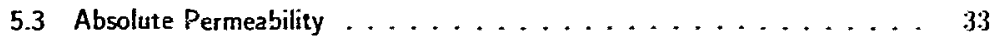

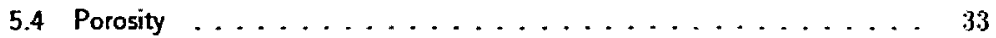

6 CONCLUSIONS $\quad 33$

7 ACKNOWLEDGEMENT

8 REFERENCES $\quad 36$

APPENDIX A. GAS RELEASE RATES

APPENDIX B. CAPILLARY PRESSLRE FOR A FRACTURE 
B.1 Function Origin $\ldots \ldots \ldots \ldots \ldots \ldots \ldots \ldots \ldots$

B.2 Parameter Adjustment . . . . . . . . . . . . 3s

B.3 Numerical Implementation . . . . . . . . . . . . . 41

B.3.1 Inverse Error Function . . . . . . . . . . . . 41

B.3.2 Arcess Procedure of Enf(x) on the Cray (See Code Listing 3) . 13

B.3.3 Treatment of Singularities . . . . . . . . . . . 13

B.3.4 Test Cases ................... $4 . \ldots$

B.3.5 $P_{c}$ vs. $S_{l}$ Functions for Different $\sigma, \bar{b} \ldots \ldots \ldots \ldots$

APPETIDIX C. STRONG INTERFERENCE RELATIVE PERMEABILITY FUNCTIONS 48

D CODE LISTINGS

D.1 FORT RAN Source Code for Calculating Fracture Cap:lary Pressure (Version 1) . . . . . . . . . . . . . . . . 50

D.2 FORT RAN Source Code for Calculating Fracture Capillary Pressure (Version 2). . . . . . . . . . . . . . . . 52

D.3 Access Procedure for Inverse Error Function from IMSLSFU N Library. $\quad 54$

D.4 Coding for Identifying the Position of the Gas Saturation Front. . . . 55

D.5 Coding for Relative Permeability Function with Strong Phase interference. 57

D.6 Sample TOUGH2-Input File for Reference Case. . . . . . . . . . 59 


\section{List of Tables}

1 Parameters for the Reference Case. ................. 4

2 Sensitivity Studies. . . . . . . . . . . . . . . .

3 Results of Sensitivity Studies. ................. 32

4 Behavior of Fracture Capillary Pressure for (a) $S_{l} \rightarrow 0$. (b) $S_{l} \rightarrow 1$. . 4

5 Numerical Check of Fracture Capillary Pressure Function $(\beta=-.3341 ; \sigma=$ $43 ; \bar{b}=.2838(\mu \mathrm{m}) \ldots \ldots \ldots \ldots \ldots . \ldots \ldots 4_{4}$ 


\section{List of Figures}

1 Schematic of Flow System for Gas Migration Studies. . . . . . . . . . 3

2 Capillary Pressure of Rough-Walled Fractures with Log-Normal Aperture Distribution. . . . . . . . . . . . . . . . 6

3 Two-Phase Relative Permeability Curves after Corey (1954) . . . . . . I I

4 Simulated Gas Saturation in Injection Grid Block for Reference Case. . . S

5 Simulated Gas Pressure in Injection Grid Block for Reference Case. . . . 9

6 Simulated Gas Saturation Profiles for the Reference Case at Different Times. 10

7 Gas Pressure Profiles for the Reference Case at Different Times. . . . . 12

8 Simulated Advance of the Gas Front for the Reference Case. . . . . . 13

9 Simulated Rate of Liquid Outflow at the Top of the Column. . . . . . 1.1

10 Capillary Pressure Functions Used in Sensitivity Studies. . . . . . . . 16

11 Sensitivity of Gas Pressures in Injection Block to Variations in Capillary Pressure. . . . . . . . . . . . . . . . 1t

12 Sensitivity of Gas Saturation in Injection Block to Variations in Capillary Pressure. . . . . . . . . . . . . . . . Is

13 Advance of Gas Displacement Front for Different Capillary Pressures. . . 19

14 Grant's Relative Permeabilities Compared with Corey's Curves Used for the Reference Case. . . . . . . . . . . . . . . 20

15 Relative Permeabilities with Strong Phase Interference, Compared with Corey's Curves. Note the Logarithmic Scale. . . . . . . . . . . . 21

16 Sensitivity of Gas Pressures in Injection Block to Variations in Relative Permeability. . . . . . . . . . . . . . . . 22

17 Sensitivity of Gas Saturations in Injection Block to Variations in Relative Permeability. . . . . . . . . . . . . . . . . 
18 Sensitivity of Gas Front Advance to Variations in Relative Permeability. .

19 Sensitivity of Gas Pressures in Injection Block to Variations in Absolute Permeability. . . . . . . . . . . . . . 20

20 Sensitivity of Gas Front Advance to Variations in Absolute Permeability. . 27

21 Sensitivity of Gas Saturations in Injection Block to Variations in Absolute Permeability. . . . . . . . . . . . . . . . 28

22 Sensitivity of Gas Saturations in Injection Block to Variations in Porosity. 29?

23 Sensitivity of Gas Pressures in Injection Block to Variations in Porosity. . 30

24 Sensitivity of Gas Front Advance to Variations in Porosity. . . . . . . 31

25 Fracture Capillary Pressures for Log-Normal Aperture Distribution. . . . 39

26 Capiliary Pressure Functions for Three Common Geologic Nedia (From Wiborgh et. al., 1986). . . . . . . . . . . . . 10

27 The Error Function. . . . . . . . . . . . . . . . . 42

28 Fracture Capillary Pressures for Different Values of Variance $\sigma . \ldots 47$ 


\section{Index}

$\tilde{b}$

Mean aperture

$b_{p}$

Cut-off aperture

erf

Error function

erfi

Inverse error function

$k_{r_{g}}$

Gas relarive permeability

$\mathrm{si}_{r_{I}}$

Liquid relative permeability

m

Moles per molecule

M

Molecular weight

$P_{c}$

Capillary pressure

$P_{g}$

Gas pressure

$P_{l}$

Liquid pressure

$Q_{3}$

Gas injection rate

$R$

Universal gas constant

$S_{1}$

Liquid Saturation

$S_{i}$

Lig̣uid in educible saturation
$S_{s r}$

Gas irreducible saturation

$V_{g}$

Gas volume

$\sigma$

Variance

$\gamma$

Surface tensiol. 


\title{
Sensitivity Studies on Parameters Affecting Gas Release from an Underground Rock Cavern
}

\author{
Erika Schlueter and Karsten Pruess
}

January 1990

\begin{abstract}
A series of numerical simulation experiments is performed to quantify the effects of the release and migration of non-condensible gas in water- saturated fractured rock formations. The relative importance of multiphase parameters such as relative permeability, capillary pressure, intrinsic Dermeability, and porosity on system behavior is studied.
\end{abstract}

\section{INTRODUCTION}

The Nationale Genossenschaft für die Lagerung radioaktiver Abfaelle (NAGRA) of Switzerland is studying the feasibility of disposing low and intermediate level nuclear wastes in a geologic repository. The corrosion of metals and microbial degradation of the organic materials from such a repository are expected to generate large amounts of gas, the main constituent being hydrogen, with minor amounts of methane, carbon dioxide, and others, (Wiborgh et.al., 1986). From the data given by Wiborgh, et.al., it can be estimated that due to the expected gas release pressures in the repository may rise to several hundred bars, unless proper venting is installed. Gas production and migration may also infiuence the transport of soluble species in groundwater, through displacement of water by the gas front, and/or a mixed flow of contaminated water and gas.

In a previous study two reference cases were developed for evaluating effects of gas release and migration at a potential nuclear waste repository site at Oberbauenstock (Pruess, 1989). The reference cases used alternative conceptualizations of the rock mass as a porous and a fractured-porous medium, respectively, and employed 'best estimates' for the hydrologic parameters applicable at the site. Many important parameters are not well known, however, leading to corsiderable uncertainty in expected behavior. The present study attempts a broader and more systematic evaluation of gas release effects. 
We consider an idealized geometric model of the flow system that represents the conditions encountered at Oberbauenstock in a schematic way. Flow effects from gas release are investigated undei a variety of conditions of relative permeability, capillary pressure, intrinsic permeability. and porosity. The numerical simulations are performed with the multiphase numerical simulator TOUGH2 using its air component to model the gas behavior (Pruess, 1987,1990).

\section{MODEL DOMAIN AND COMPUTATIONAL GRID}

It is assumed that gas release occurs uniformly over a large area. Ignoring regional-scale boundary effects, the flow system is taken to be a one- dimensional vertical column of I $\mathrm{m}^{2}$ cross-sectional area and $100 \mathrm{~m}$ height (Figure 1). The column is assumed to be water-saturated initially with a hydrostatic gradient, so that initial pressure at the bottom is approximately 10 bars. Pressure is maintained at ambient $P=1$ bar at the top, while gas is injected at the bottom of the column at a rate of $10^{-8} \mathrm{~kg} / \mathrm{s} \times \mathrm{m}^{2}$ (Appendix A). For numerical simulation, the section is subdivided into 40 elements spaced at $2.5 \mathrm{~m}$. The parameters for the reference case are similar to those used in the porous medium case of Pruess (1989), except that we include a non-zero capillary pressure (Table 1, and Appendix B). The parameter variations considered in the sensitivity studies are summarized in Table

2 (Cases 1-8). A sample input file for TOUGH2 is shown in code listing 6 . Note that, because of the one-dimensional model used in this report, we cannot address issues of formation heterogeneities and (viscous or gravitational) flow instabilities.

\section{SYSTEM BEHAVIOR FOR THE REFERENCE CASE}

The gas injected into the porous medium initially dissolves in the water that is present in the injection grid block. However, gas solubility is soon exceeded and a free gas phase forms (Figure 4). Further gas release leads to an increase in gas saturation accompanied by pressurization, as water is displaced by the growing gas bubble (Fig.5). At approximately $6 \times 10^{6} s$ ( 80 days), gas saturation reaches the irreducible limit of $5 \%$, and begins to flow upward. A gas-water displacement front then migrates up the column (Figure 6). Note that the pressure gradient is smaller behind the displacement front than ahead of 


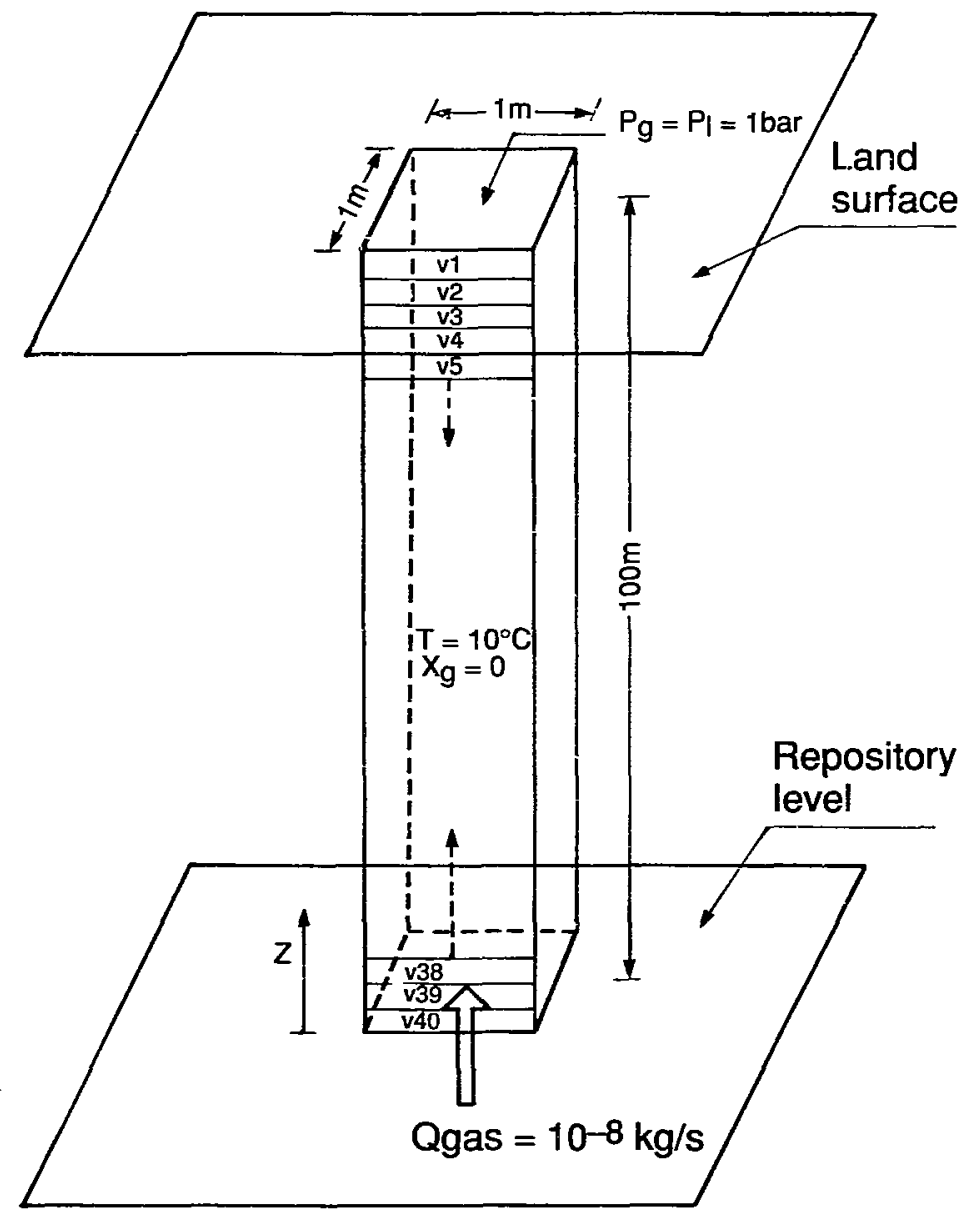

XBL 902-6236

Figure 1: Schematic of Flow System for Gas Migration Studies. 
TABLE 1. Parameters for the Reference Case

Formation Parameters:

$\frac{\text { permeability }}{10^{-17} m^{2}}$

$\frac{\text { porosity }}{1 \%}$

$\frac{\text { compressibility }}{10^{-10} P a^{-1}}$

Relative Permeability:

(Corey's Curves; see Fig.3)

liquid phase:

$$
\begin{aligned}
& k_{r l}=\left[S^{*}\right]^{4} \\
& k_{r g}=\left(1-S^{*}\right)^{2}\left(1-\left[S^{*}\right]^{2}\right) \\
& S^{*}=\left(S_{l}-S_{l r}\right) /\left(1-S_{l r}-S_{g r}\right) \\
& S_{l r}=0.3 \\
& S_{g r}=0.05
\end{aligned}
$$

gas phase:

\section{Capillary Pressure:}

Fracture capillary pressure as derived for a lognormal aperiure distribution by Pruess and Tsang, 1989; see Appendix B, and Fig.2.

$$
\begin{aligned}
& P_{c}=2_{\gamma} 10^{\left(\sqrt{2} \sigma e r f i\left(1-2 S_{l}\right)-3\right)} \\
& \gamma=0.073 \mathrm{~N} / \mathrm{m} \\
& \beta=-0.3344 \\
& \sigma=0.43 \\
& b_{a v e}=.2836 \mu \mathrm{m}
\end{aligned}
$$

\section{Boundary Conditions:}

bottom:

top:

\section{Initial Conditions:} temperature:

pressure:

gas saturation:
Air Injection Rate: $10^{-8} \mathrm{~kg} / \mathrm{s}$

$P_{g}=P_{l}=10^{5} P a \equiv 1 b a r$

$\left(P_{\text {cap }}=0\right)$ $10^{\circ} C$

hydrostatic pressure gradient

no air present

Table I: Parameters for the Reference Case. 
TAbLE 2. Sensitivity Studies

\section{CASE}

1

2

3

4

5

6

7

8

\section{VARIATION}

stronger capillary pressure, with air entry effects

(Narasimhan et.al., 1978; see Fig.10)

parameters: $I C P=3, P_{o}=5 \times 10^{5} \mathrm{~Pa}$,

$S_{l \mathrm{r}}=0.3, \nu=1.0, P_{\mathrm{e}}=2 \times 10^{5} \mathrm{~Pa}$

no capillary pressure

$P_{p}=0$

Grant's relative permeabilities (Grant, 1977; see Fig.14)

parameters: $I R P=4$;

other specifications as in reference case

strong interference relative permeabilities

(see Appendix C, and Fig.15)

parameters: $I R P=9$;

other specifications as in reference case

intrinsic permeability $k=2 \times k_{\text {ref }}$;

other specifications as in reference case

intrinsic permeability $k=0.5 \times k_{\text {ref }}$;

other specifications as in reference case

porosity $\phi=2 \times \phi_{\text {ref }}$;

other specifications as in reference case

porosity $\phi=0.5 \times \phi_{\text {ref }}$;

other specifications as in reference case

Tabie 2: Sensitivity Studies. 


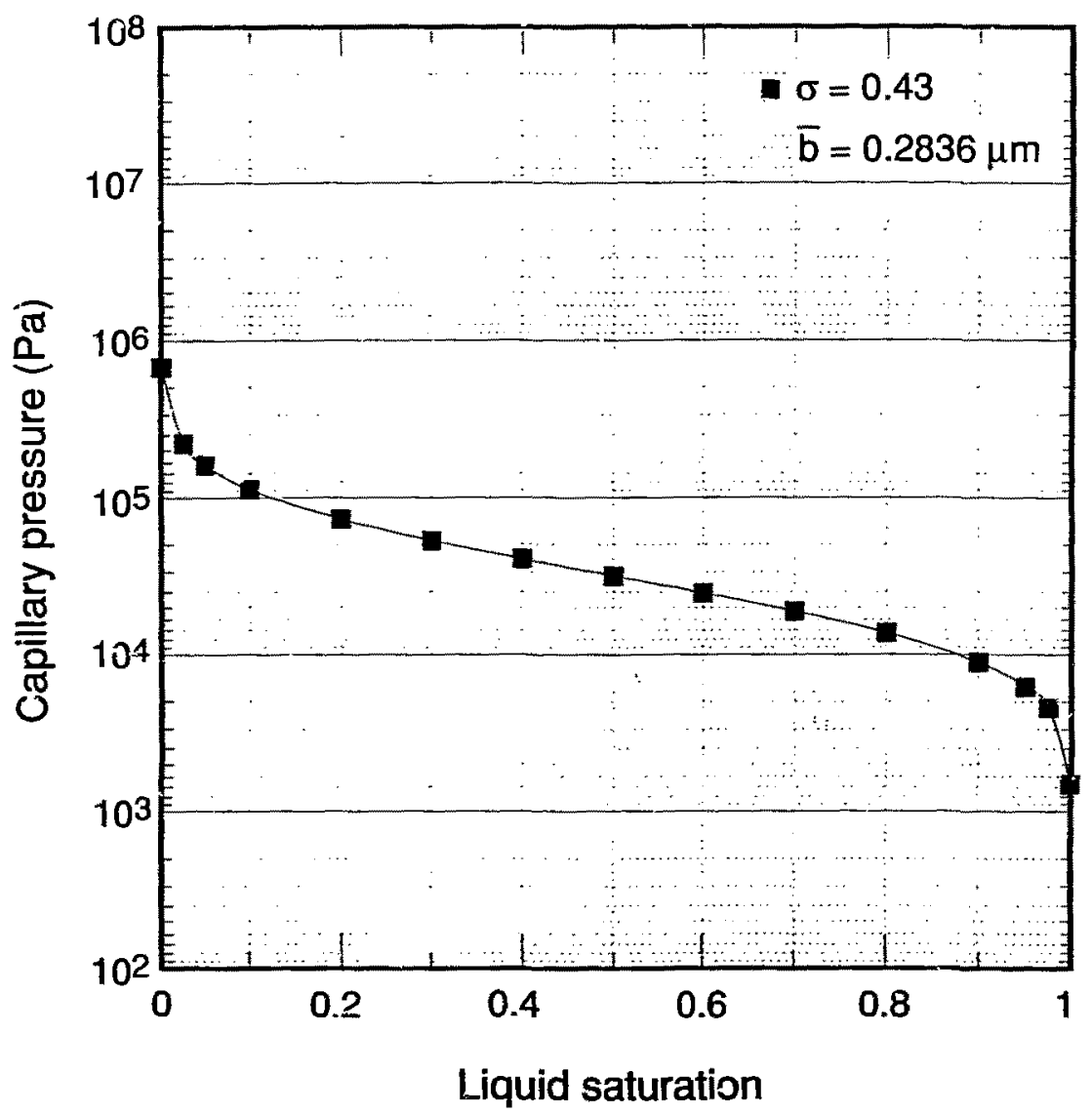

XBL 902-6237

Figuce 2. Capillary Pressure of Rough-Walled Fractures with Log-Normal Aperture Distribution. 


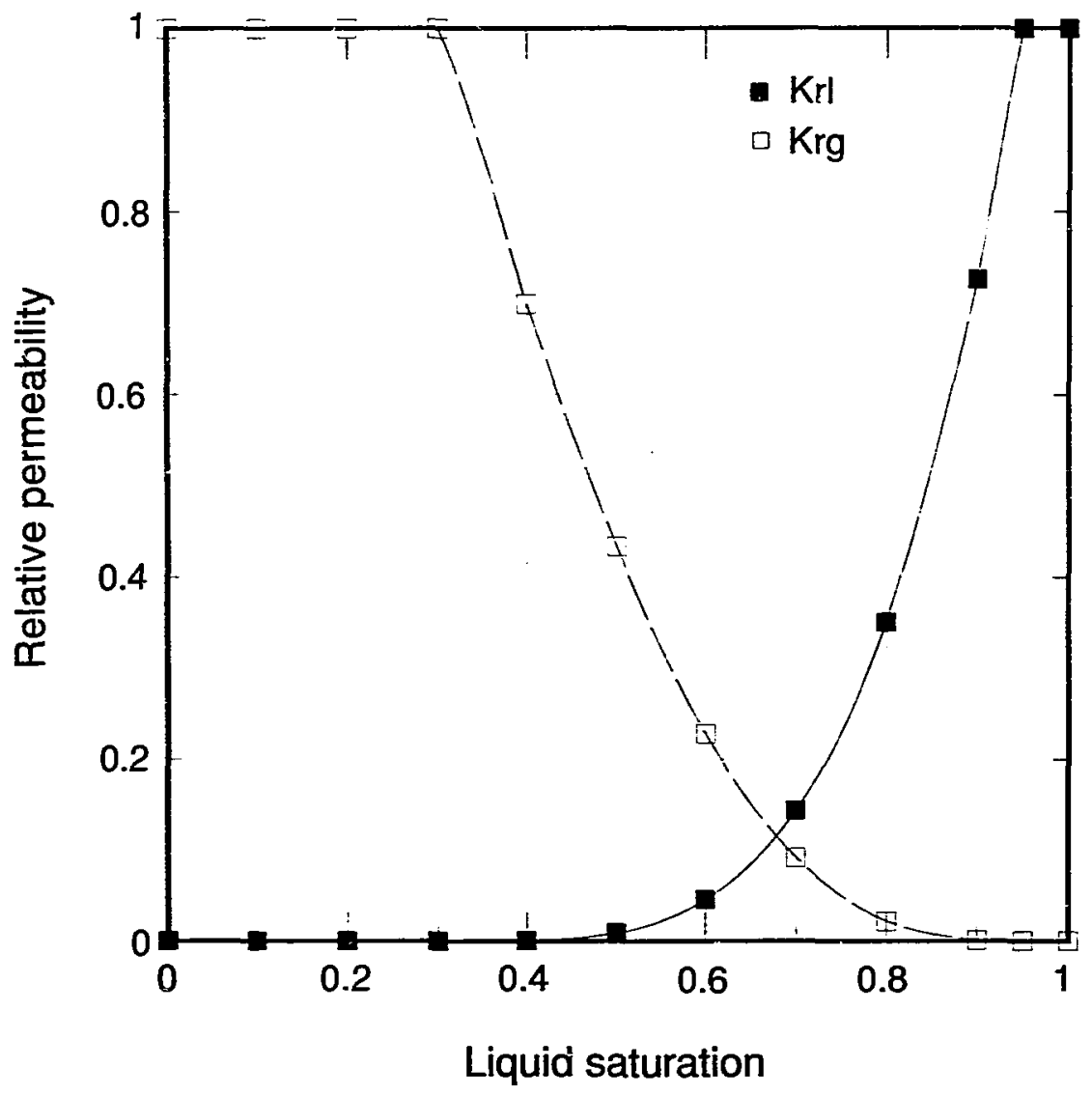

XBL 902-6:238

Figure 3: Turo-Phase Relative Permeability Curves after Corcy (1954). 


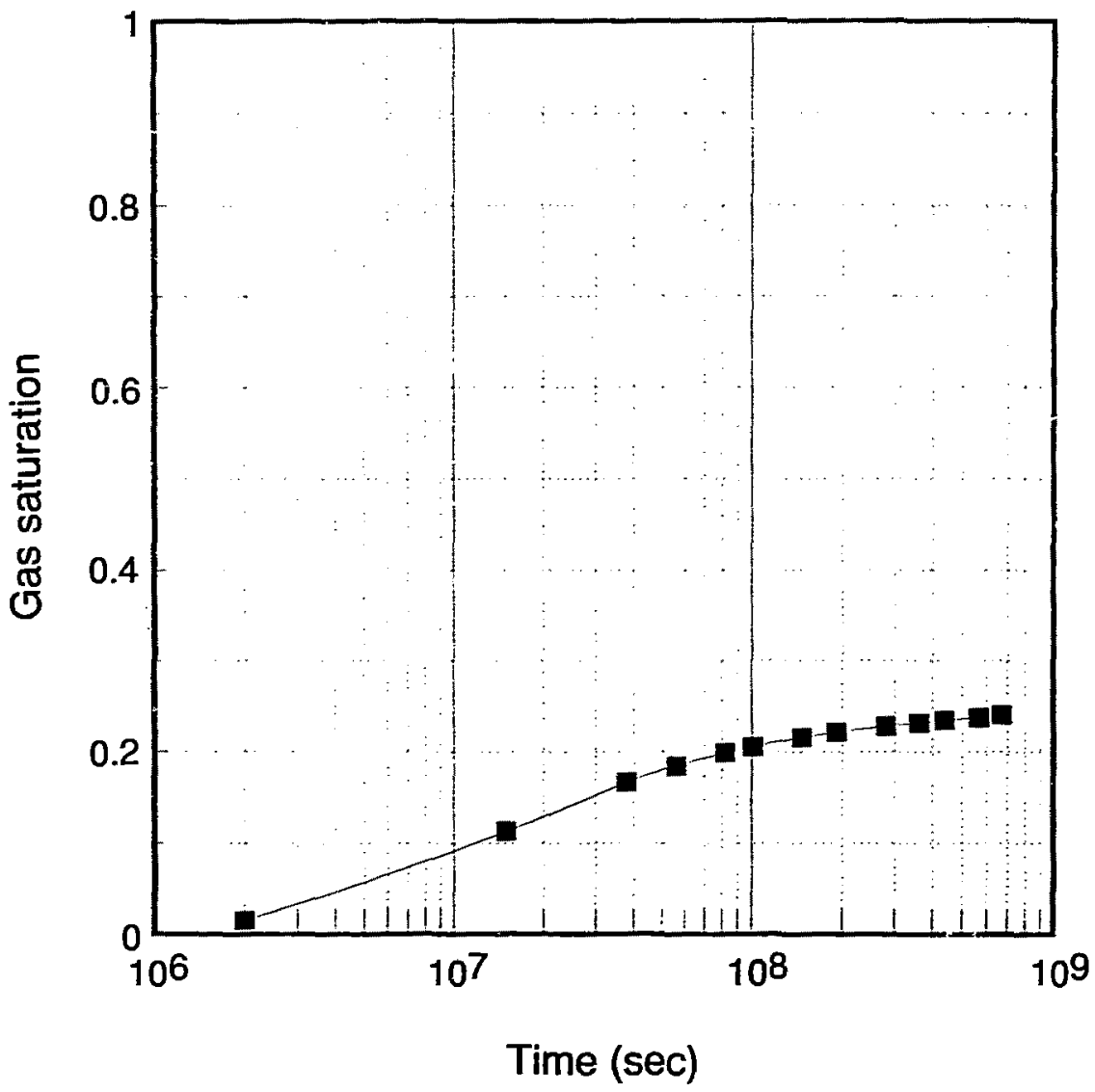

XBL 902-6239

Figure 4: Simulated Gas Saturation in Injection Grid Block for Reference Case. 


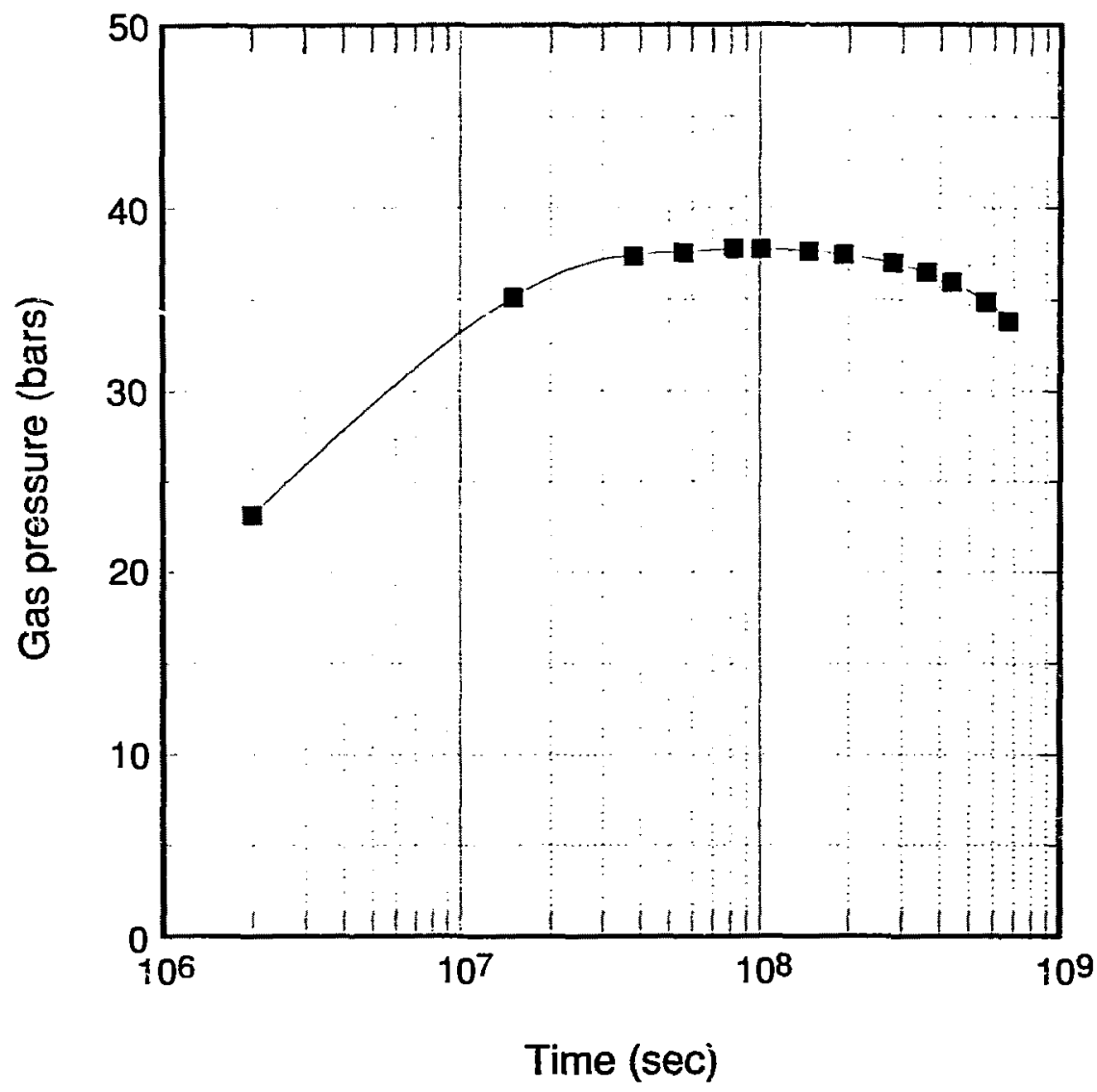

XBL 902-6242

Figure 5: Simulated Gas Pressure in Injection Grid Block for Re fercace Case. 


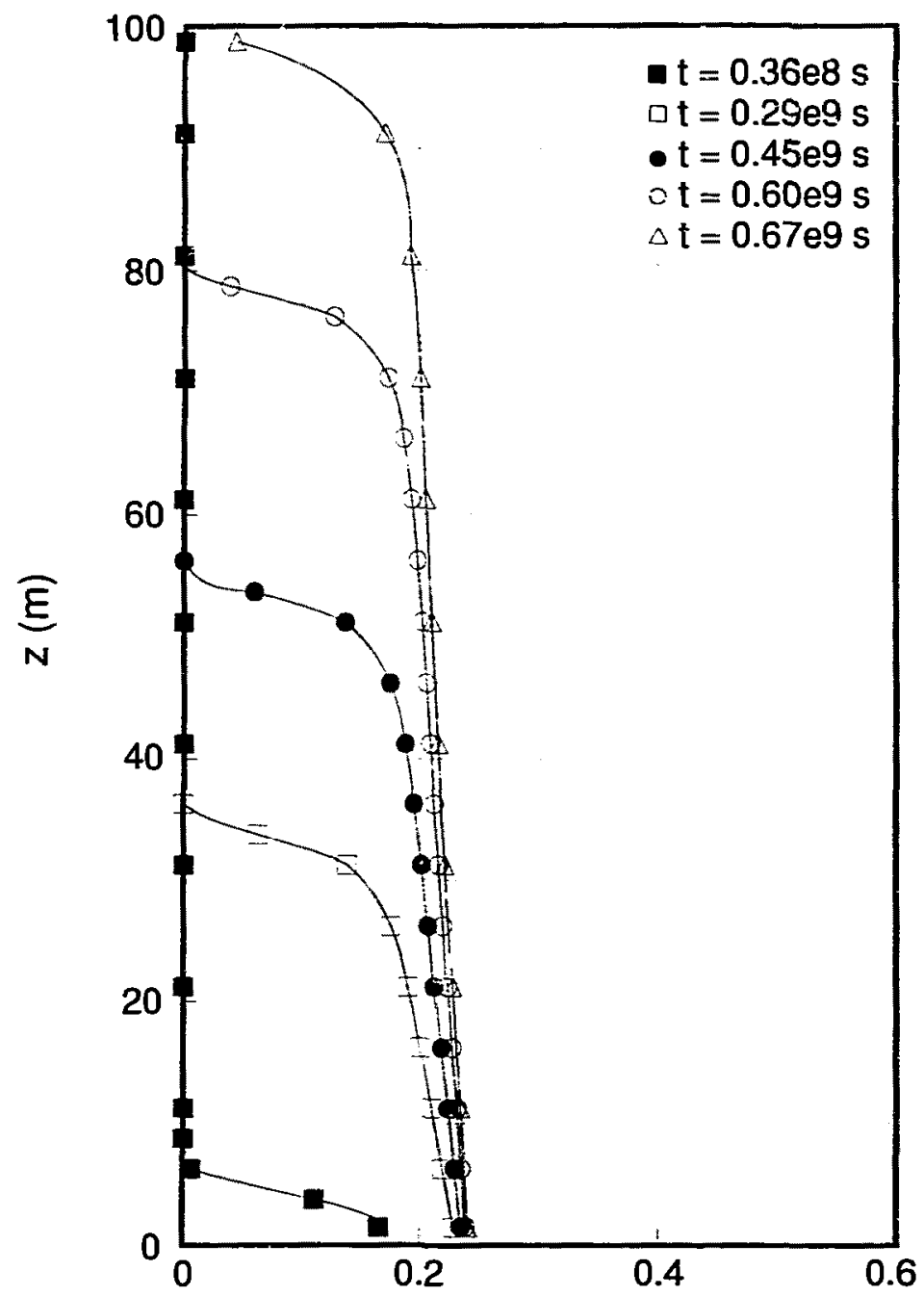

Gas saturation

Figure 6: Simulated Gas Saturation Profiles for the Reference Case at Different Times.

XBL 902-6240 
it (Figure 7), due to the much smaller viscosity of the gas phase. In the well block, gas pressure rcaches its maximum (38 bars) at about $7 \times 10^{7} \mathrm{sec}(2.2$ years) (Fig.5). Then it enters into a period of slow decline, with a continued increase in gas saturation. After $6.5 \times 10^{8} \mathrm{sec}(20.6 \mathrm{years})$ the gas reaches the top of the boundary of the flow domain (Figs.6,8). It is of interest to consider the liquid outflow at the top of the column in response to gas injection at depth (Fig.9). Due to the small compressibility of liquid water, the pressurizztion at depth is transmitted rapidly upward. Outflow at the top commences as soon as a free gas phase is formed near the injection point. The outflow rate increases rapidly at first, and more slowly after gas becomes mobile at depth $\left(\approx 6 \times 10^{\circ i} \mathrm{~s}\right)$. A quasi-steady situation with slowly increasing liquid outflow prevails until about $5 \times 10^{8} \mathrm{~s}$. Subsenguently, as the gas front approaches the top boundary, liquid outflow is enhanced from the steep pressure gradient ahead of the front (see Fig.7).

\section{SENSITIVITY STUDIES}

The hydrologic parameters that characterize fractured rock masses on a large scale are difficult to deterimine accurately in practice. It is important to evaluate the uncertainty in flow behavior that arises from the parameter uncertainty. In this sertion we present results of sensitivity studies, which examine the impact on flow behavior of variations in capillary pressures, relative permeabilities, absolute permeability, and porosity. All of these formation parameters are of course interrelated, because they all depend on the geometry of the pore space in which flow takes piace. For certain classes of permeable media, such as unconsolidated sands. quantitative relationships between some of these parameters have been found. However, due to the inherent variability of the pore geometry in natural media, there are no generally useful quantitative relationships that would apply to broad classes of media. Fo: example, one expects the permeability of a medium to generally decrease with decreasing porosity. However, in fractured media one often encounters large permeability accompanied by small porosity. Because of the strong inherent variability of flow properties of fractured rock masses, we have adopted the approach of varying the importarit parameters one at a time, independently of each other. The specifications of the sensitivity studies are given in Table 2. 


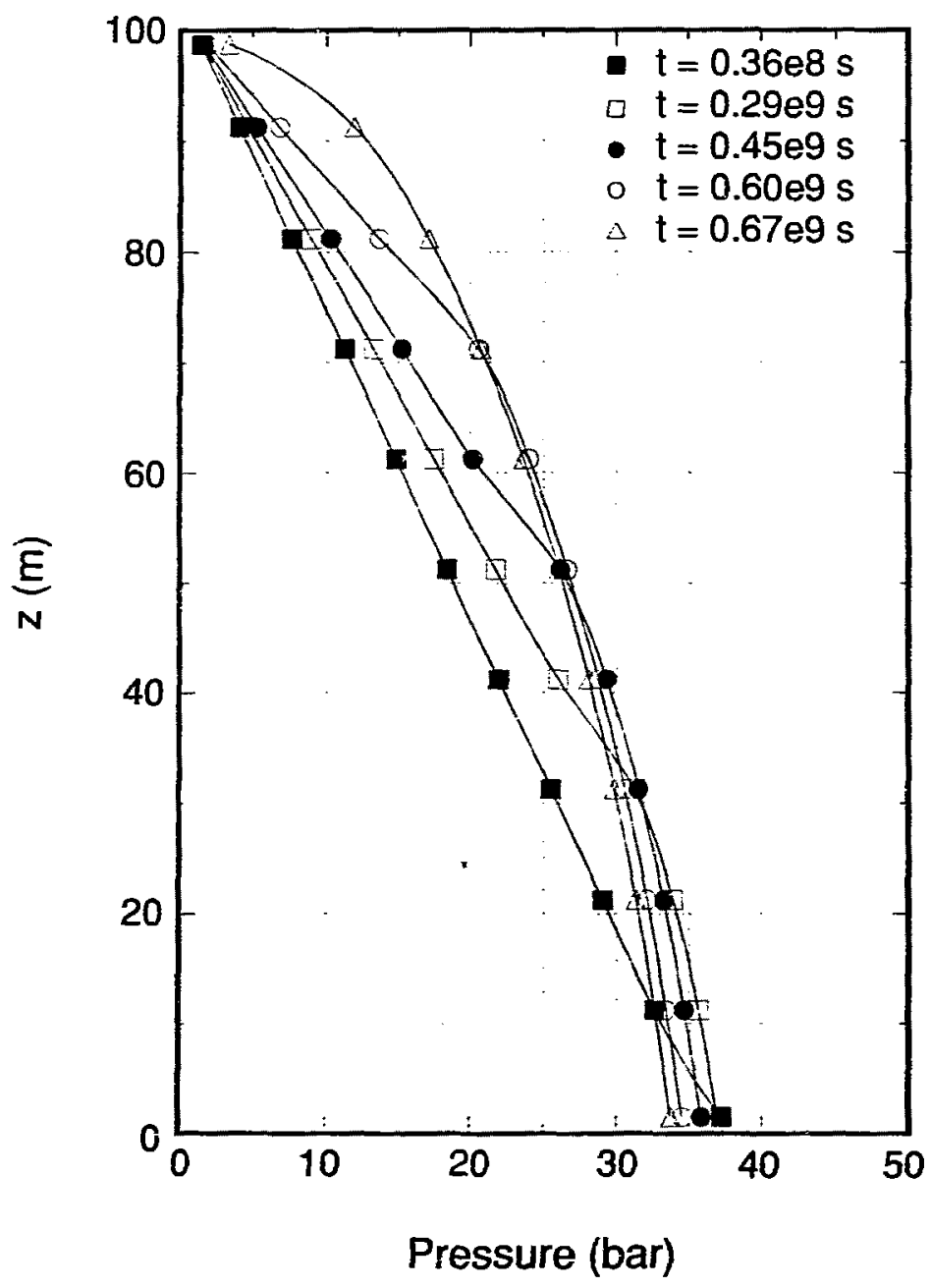

XBL 902-6241

Figure i: Gits Pressture Profles for the Reference Case at Dilferent "Times. 


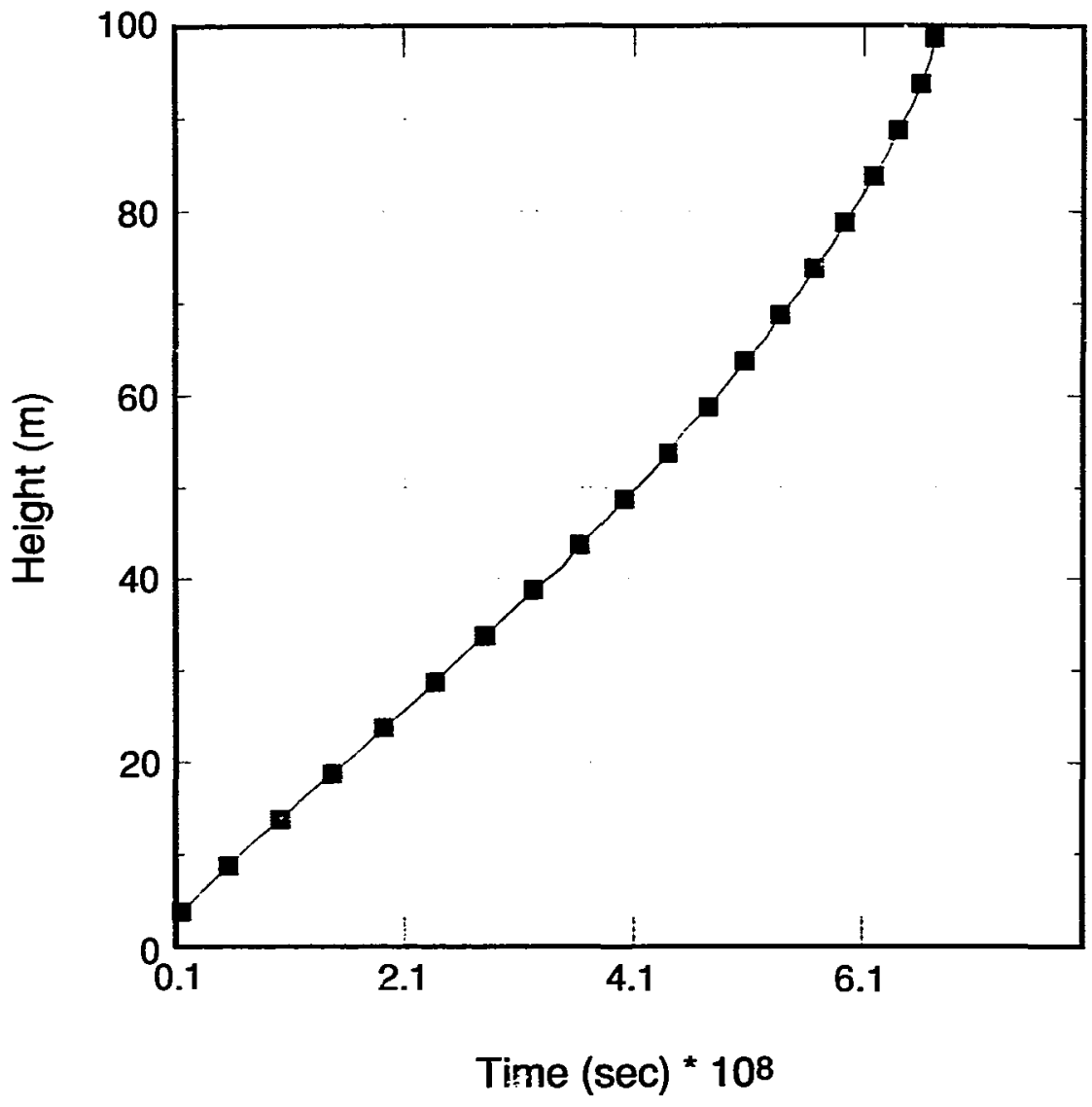

XBL 902-6243

Figure 8: Simulated Advance of the Gas Front for the Reference Case. 


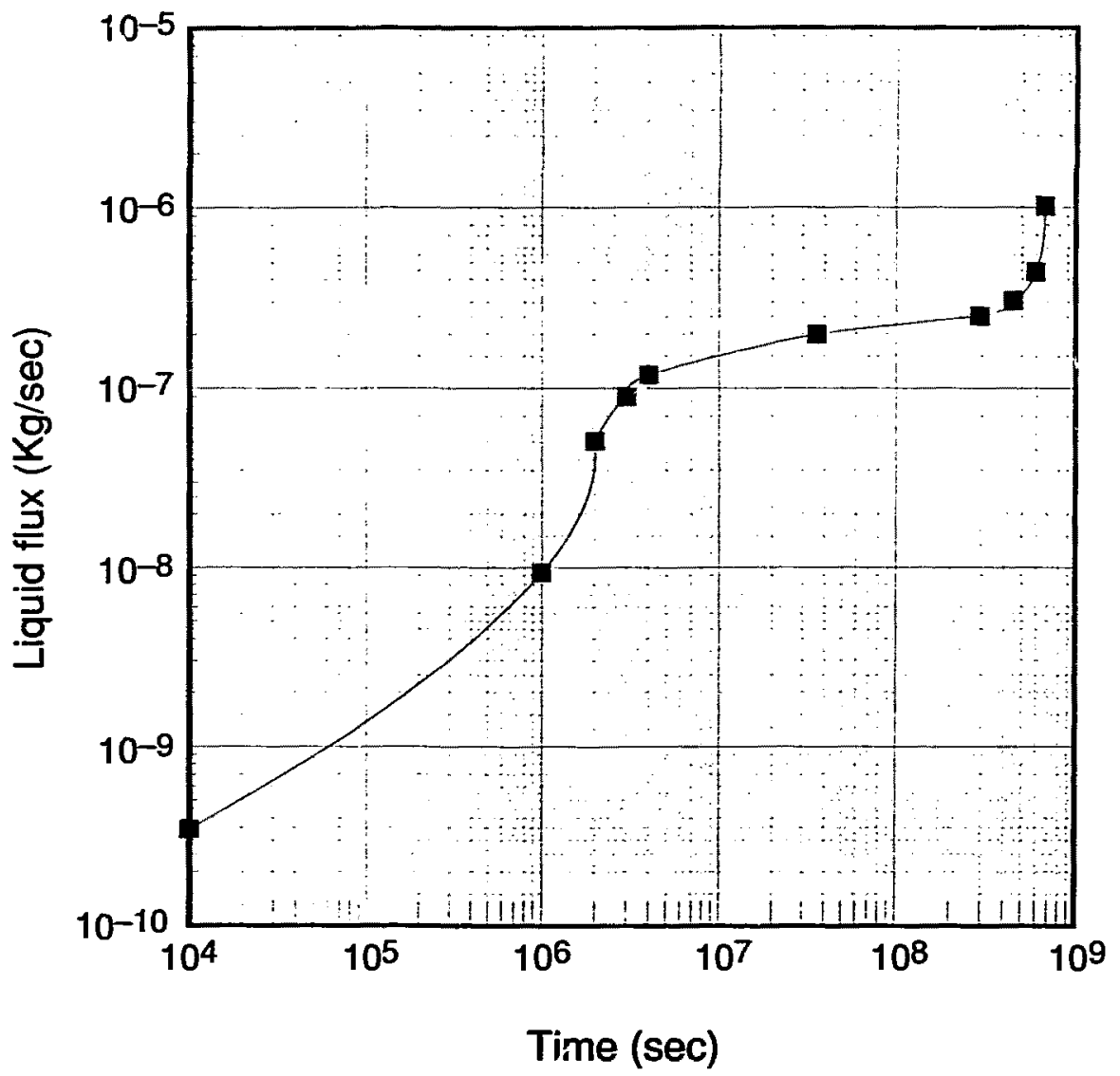

XBL 902-6244

Figure 9: Sinulated Rate of Liquid Outflow at the Top of the Column. 


\subsection{Capillary Pressure}

To explore the sensitivity of system behavior to variations in capillary pressure, Case 1 uses a stronger capillary pressure with air entry effects (Fig.10; Narasimhan et. a!., 1978). while in Case 2 capillary effects are neglected. Stronger capillary pressures accelerate and increase gas pressurization at the injection point (Fig.11), as expected, while slowing and diminishing gas saturation buildup (Fig.12). Neglect of capillary pressures has the opposite effects. Capillary pressures are generally relatively weak compared to the pressurization from viscous flow resistance, so that their impact in systems response is minot. This can also be seen from the weak retardation of the gas displacement front due to capillary effects (Fig.13).

\subsection{Relative Permeability}

There is considerable uncertainty at present about the relative permeability behavior of fractured rock masses. It has often been postulated or assumed that fracture reiative permeabilities should obey the constraint $k_{r l}=k_{r g}=1$ (Grant, 1977; Gilman and Kazemi. 1983), while recent theoretical work has suggested that phase interference in fractures may be very strong, with both $k_{r l}$ and $k_{r g}$ being small at intermediate saturations (Pruess and Tsang, 1989). Here we consider two variations on the reference case, corresponding to the conventional view of fracture relative permeabilities (Case 3; Fig.14) and the recent suggestion of strong phase interference (Case 4; Fig.15, and Appendix C), respectively. Figures 16 and 17 show that relative permeability uncertainty can have a very major effect on predicted fluid pressures and gas saturations. For the Grant curves (Case 3). gas flow is facilitated in comparison to the reference case, giving rise to smailer pressure and saturation increases. The strcag interference relative permeabilities (Case 4) on the other hand, produce a vastly stronger pressure response and much higher gas saturations. Very strong corresponding effects are seen on the advancement of the gas displacement front (Fig.18). 


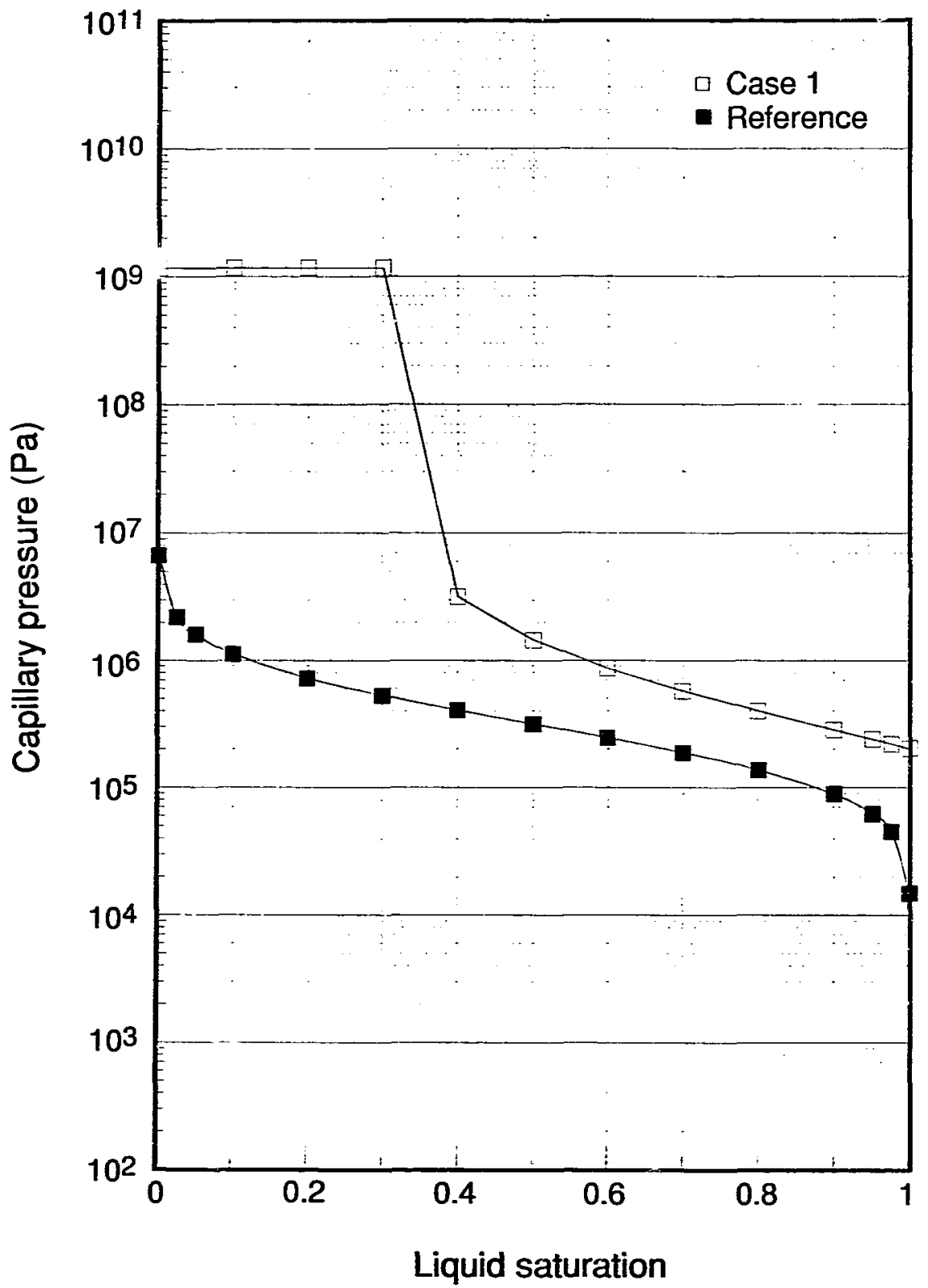

Figure 10: Capillary Pressure Functions lised in Sensitivity Studies. XBL 902-6245 


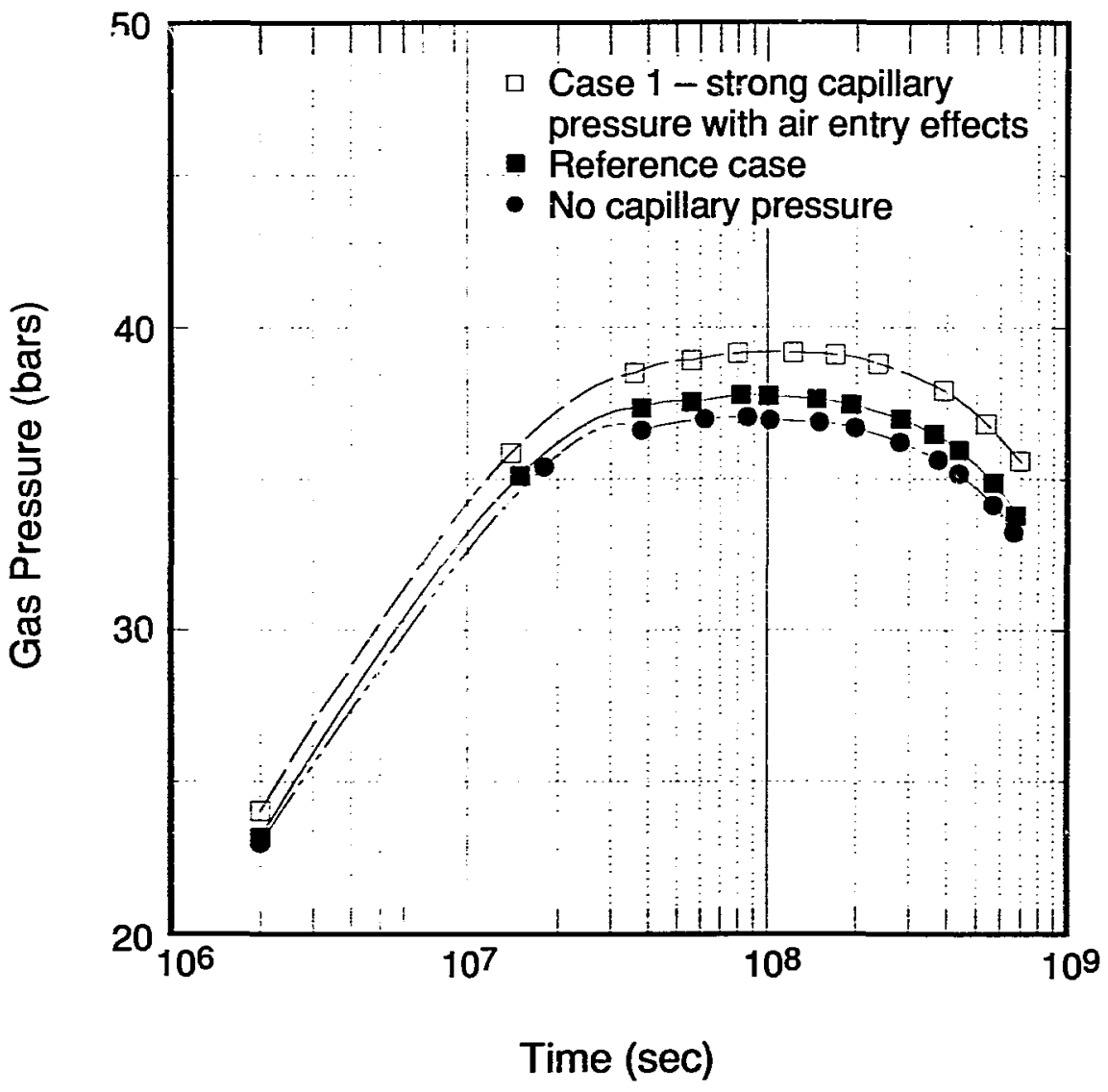

XBL 902-6246

Figure 11: Sensitivity of Gas Pressures in Injection Block to Variations in Capillary Pressure. 


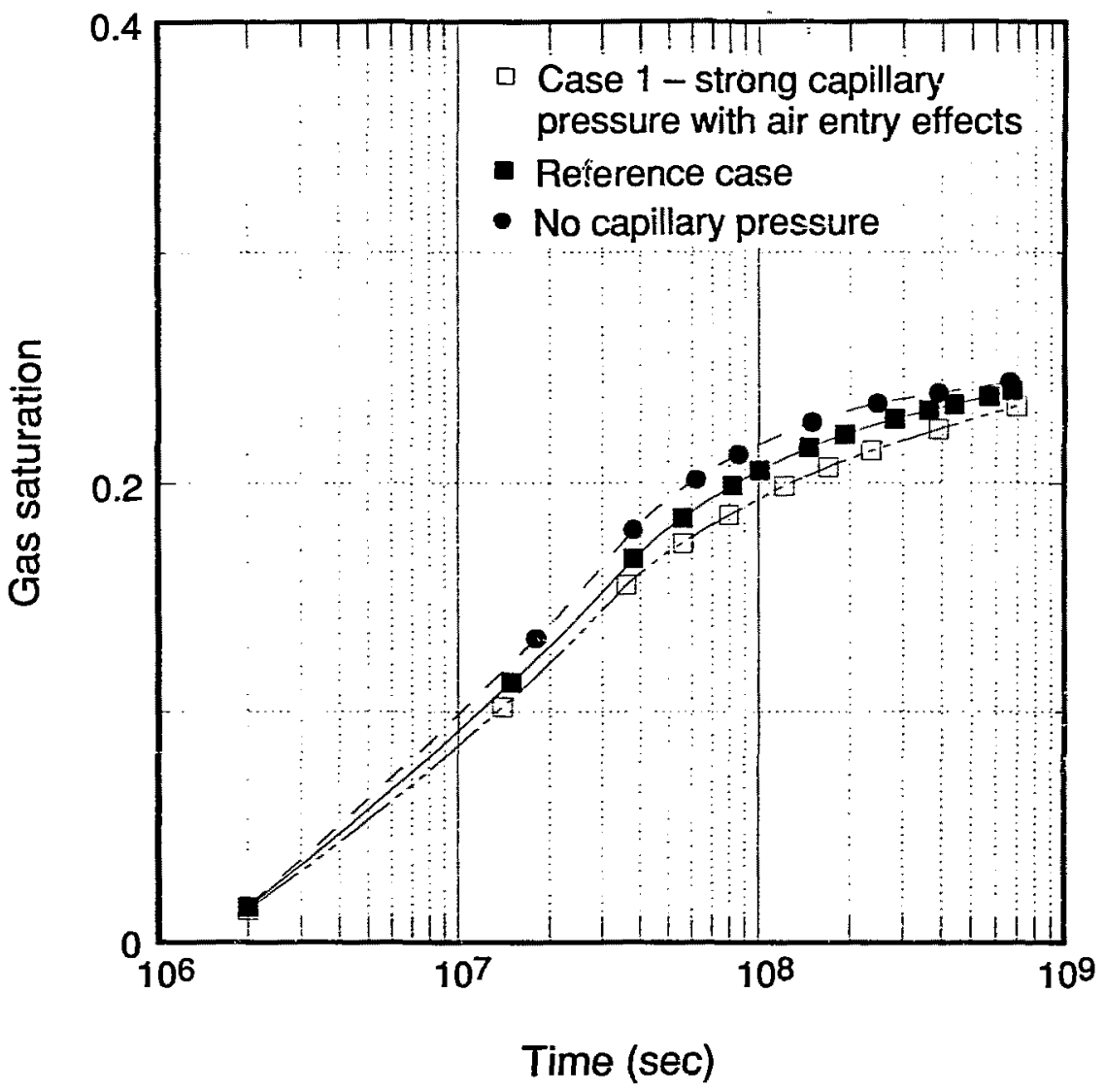

XBL 902-6247

Figure 12: Sensitivity of Gas Saturation in Injection Block to Variations in Capillary Pressure. 


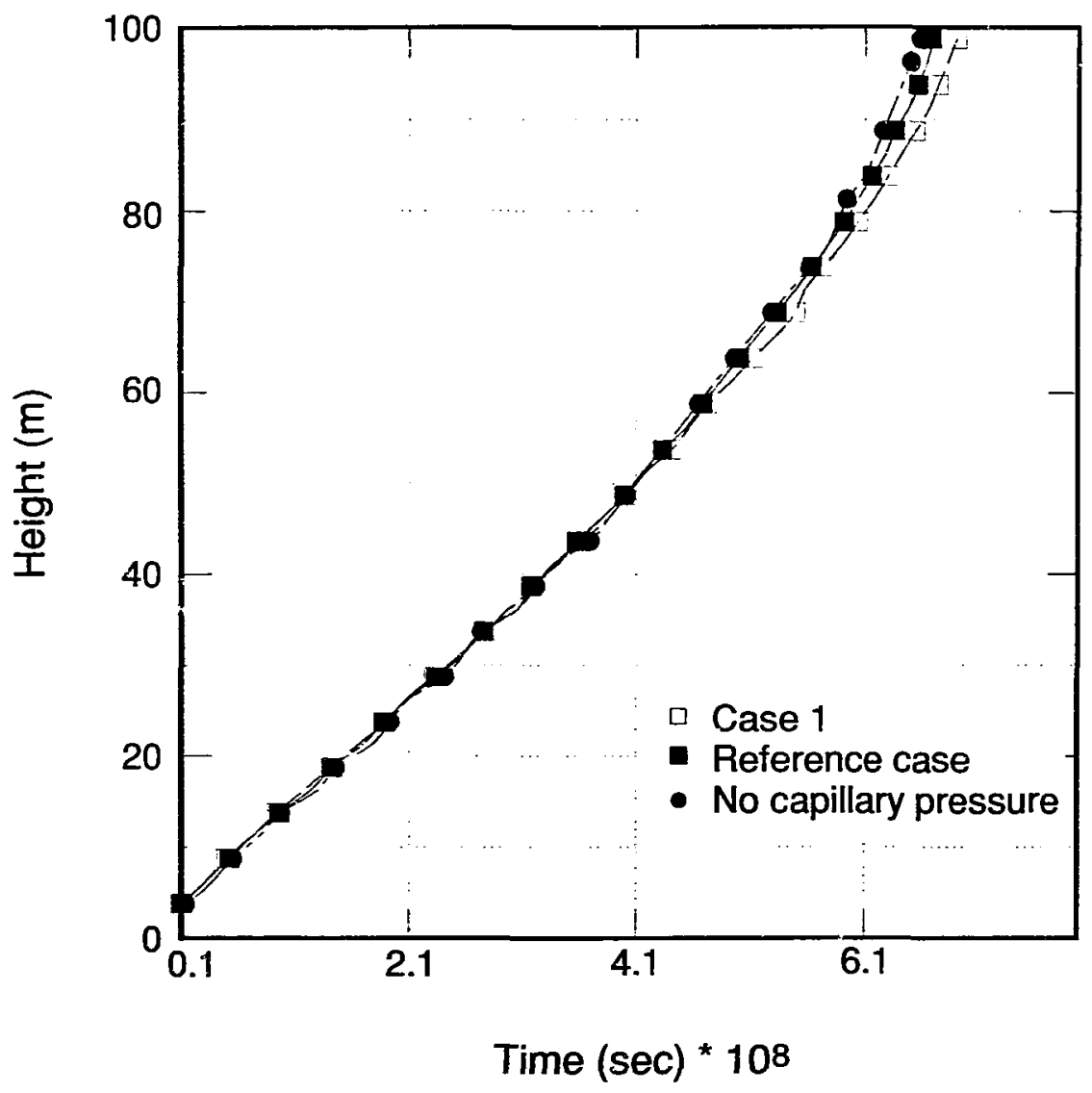

$X$ X' 902-6248

Figure 13: Advance of Gas Displacement Front for Different Capillary Pressures. 


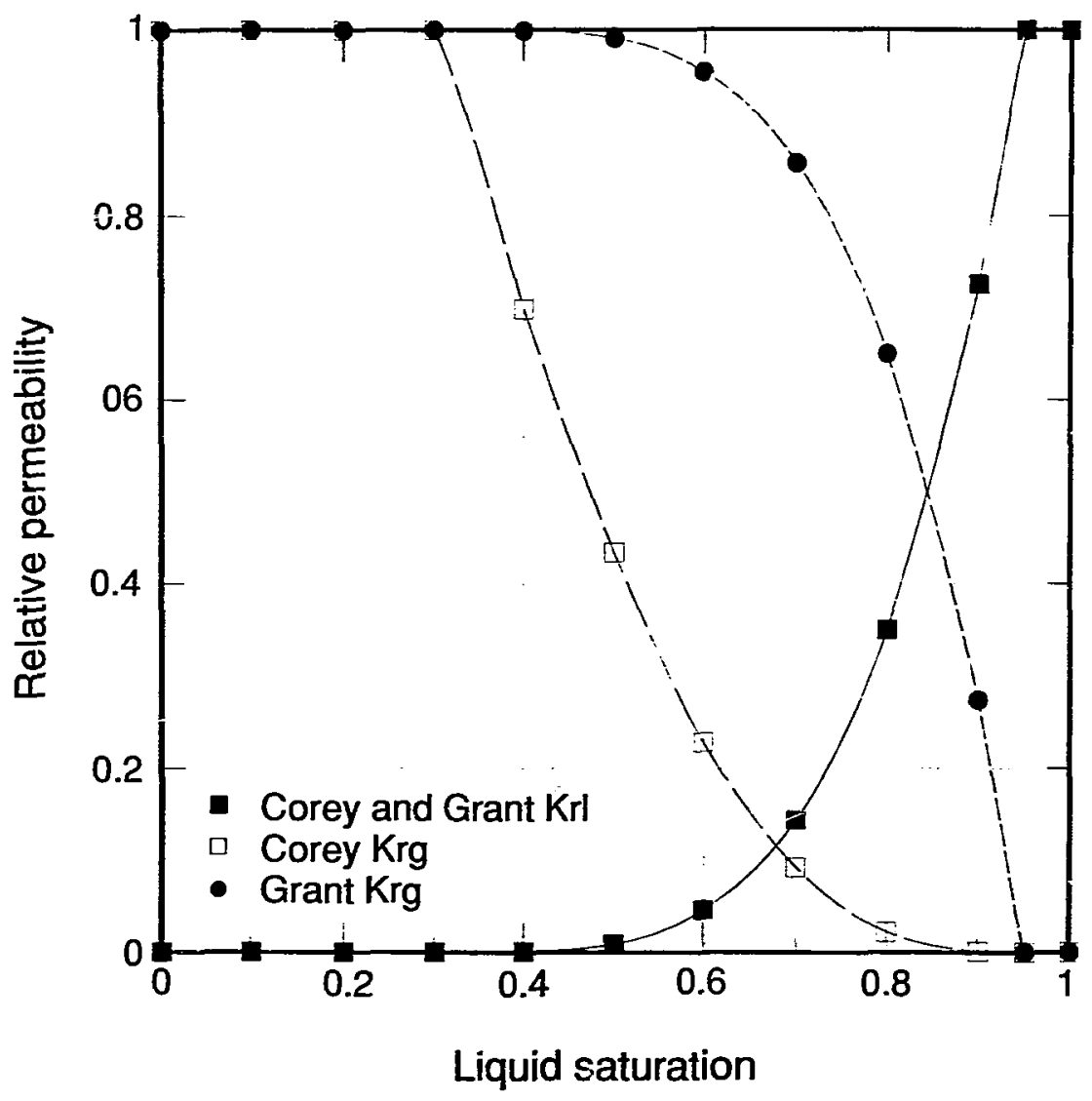

XBL 902-6249

Figure 14: Grant's Relative Permeabilities Compared with Corey's Curves lsed for the Reference Case. 


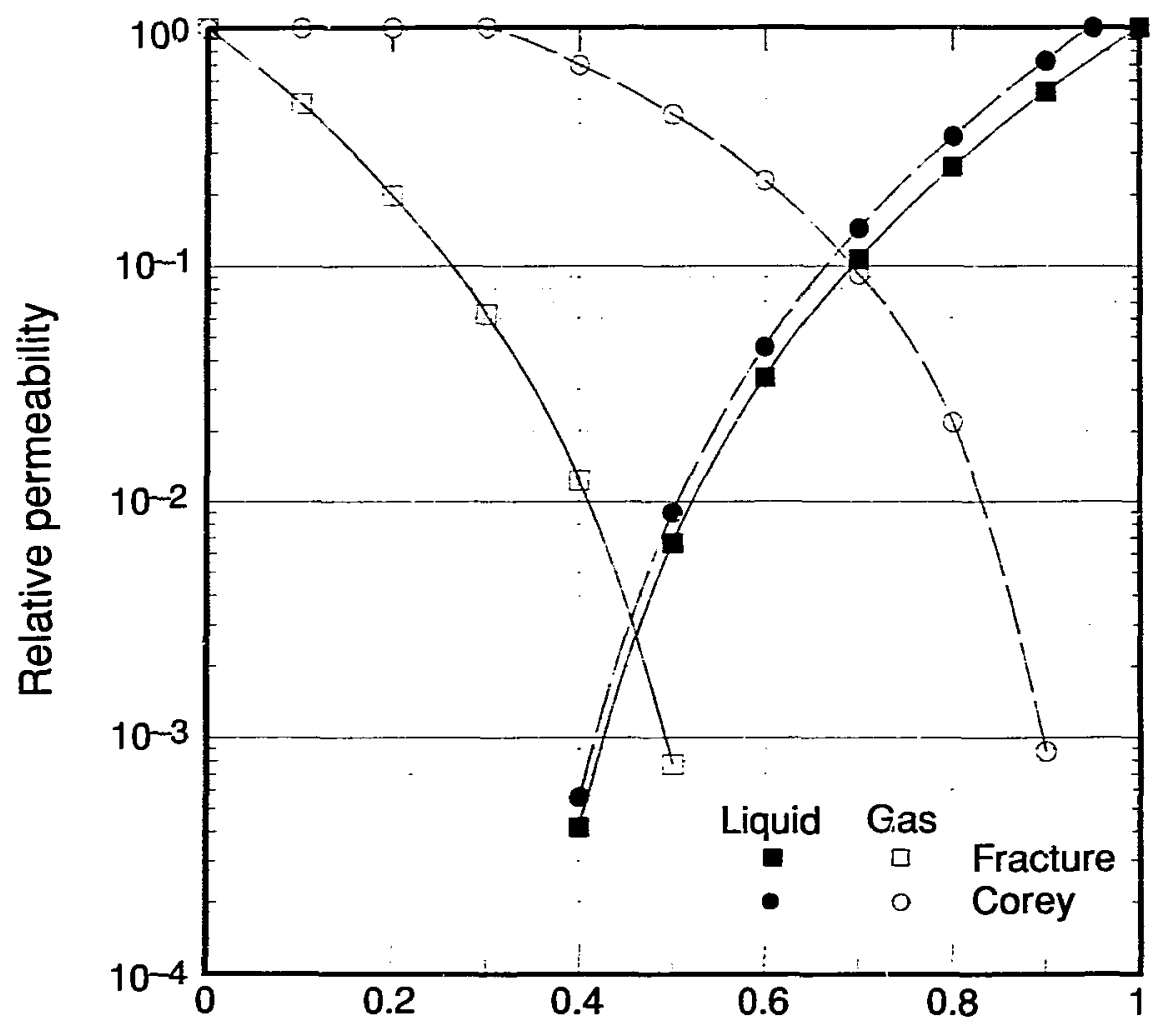

\section{Liquid saturation}

F: rure 1.5: Relative Permeabilities with Strong Phase Intrference. Compared with Corey"s ("urus Vite the Logarithmic Scale. 


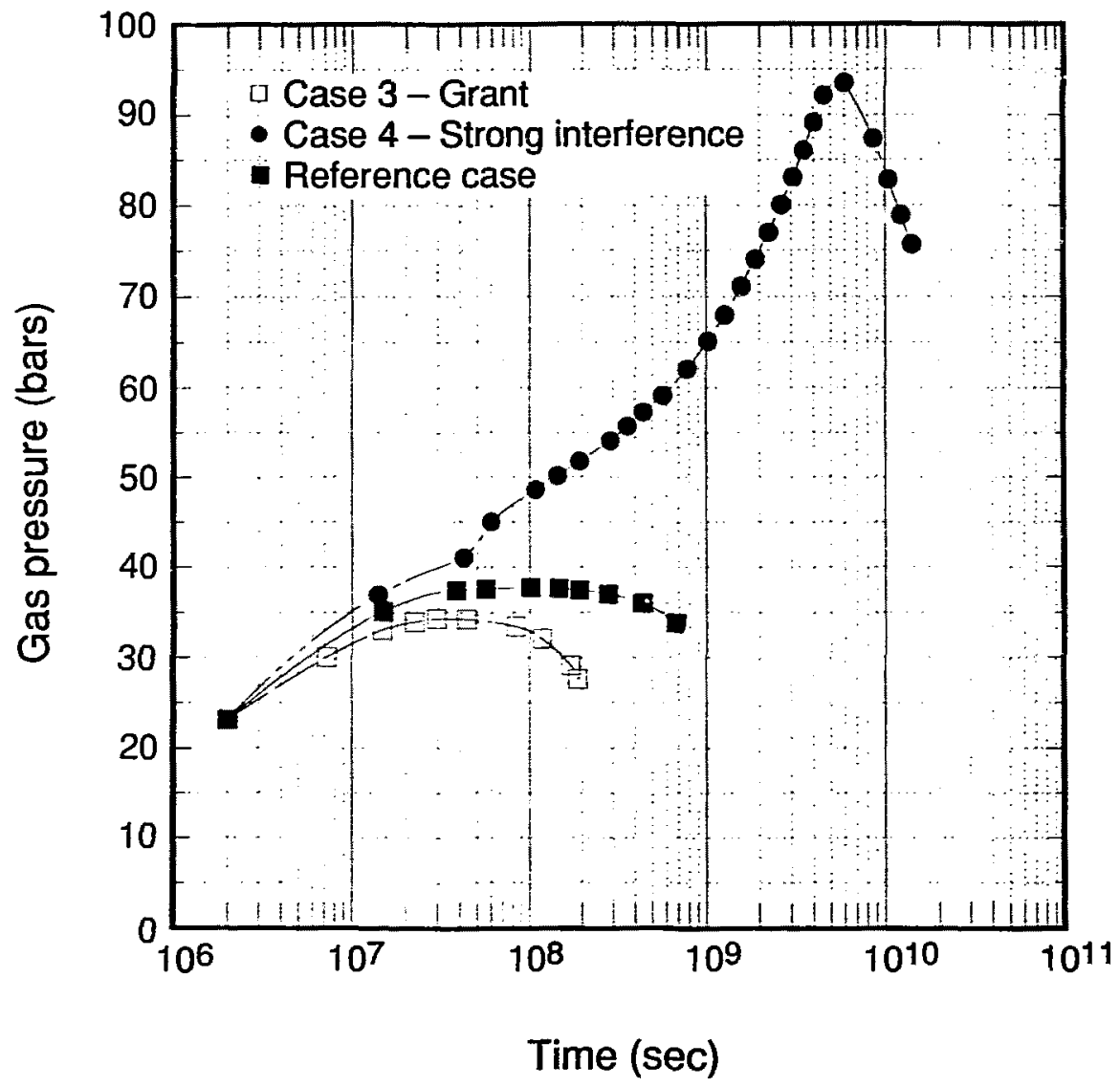

XBL 902-6251

Figure 16: Sensitivity of Gas Pressures in Injection Block to Variations in Relative Permeability. 


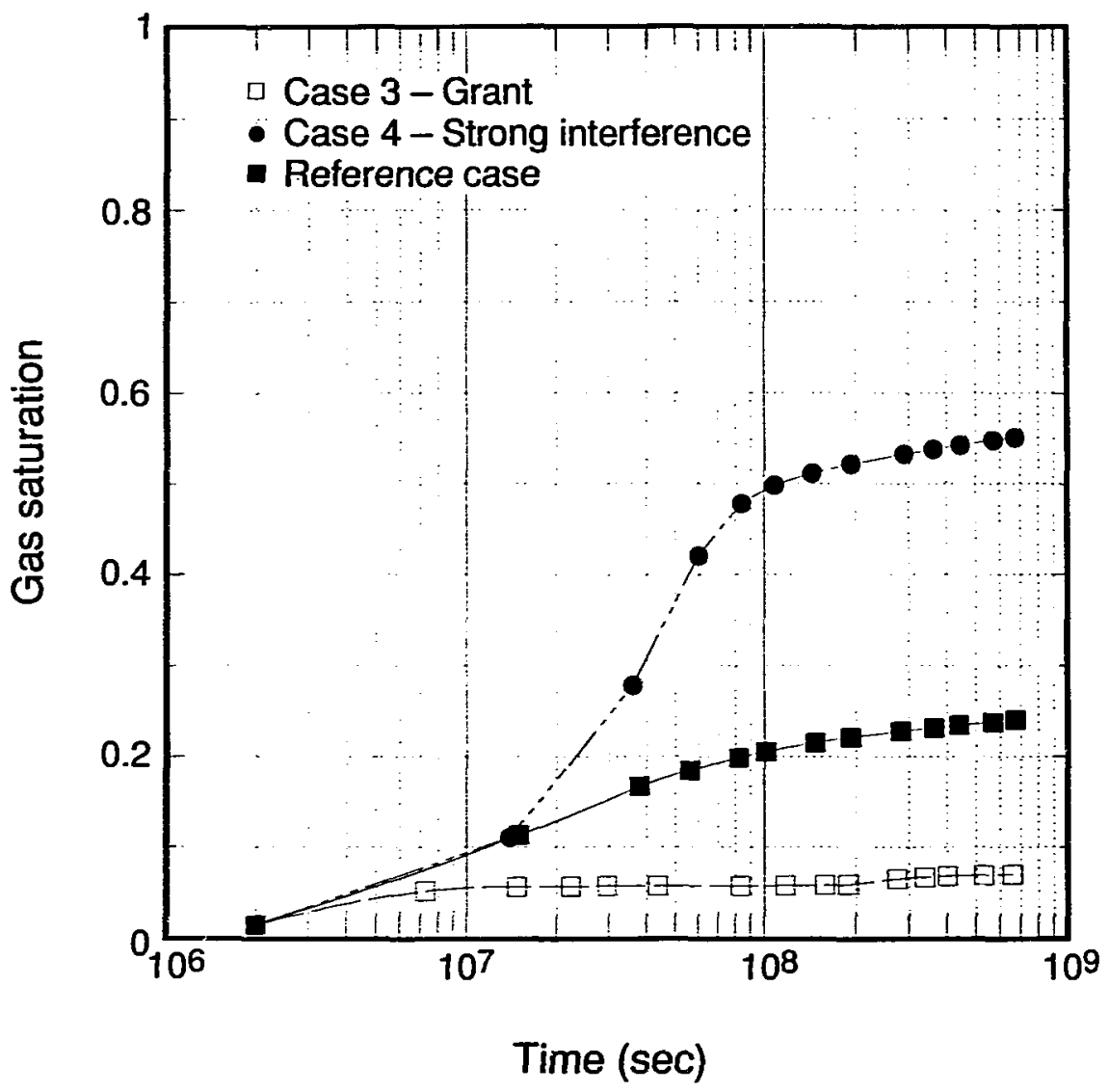

XBL 902-6252

Figure 17: Sensitivity of Gas Saturations in Injection Btock to Variations in Relative Permeability. 


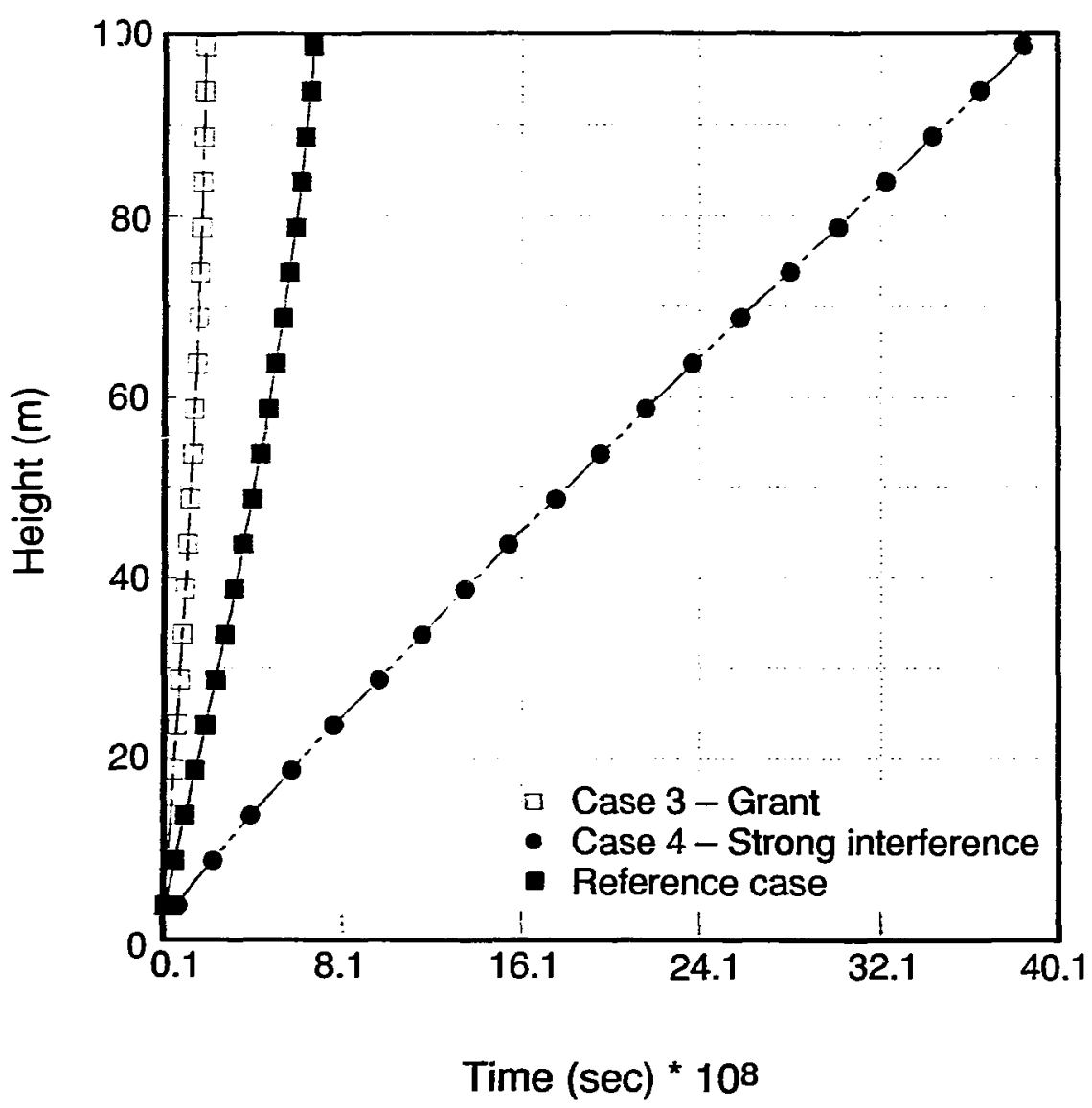

Figure 18: Sensitivity of Gas Front Mdince to Vuriations in Relative Prrmeability. XBL 902-6253 


\subsection{Absolute Permeability}

Variation of absolute permeability produces the expected effects (Figs. 19,20). Fluid pressures near the injection point increase (decrease) when permeability is diminished (enhanced). For higher permeability the advance of the gas front is more rapid. It is interesting to note that permeability effects on gas saturation are rather minor (Fig. 21).

\subsection{Porosity}

Effects from porosity variations are somewhat complemertary to those from permeability variations (Figs. 22,23). Gas saturation near the injection point increases more rapidly and to higher values for smaller porosity, due to the smaller available pore volume. Pressure response is also more rapid for smaller porosity, but the pressure level maintained during the advancement of the gas displacement front up the column is little affected by porosity. The rate at which the gas front advances is approximately inversely proportional to porosity (Fig.24).

\section{SUMMARY OF MULTPHASE EFFECTS}

For the particular process considered here, i.e., displacement of water by gas, the impacts of system parameters on flow behavior can be summarized as follows: (See Table 3)

\subsection{Capillary Pressure}

Its effects were found to be generally weak, becatise typical capillary pressures are only of the order of a few bars, while pressure increase from viscous flow resistance in the problem considered here is typically a few tens of bars. Increased capillary pressures will tend to cause increases in gas phase pressurization, while diminishing and slowing gas saturation buildup. 


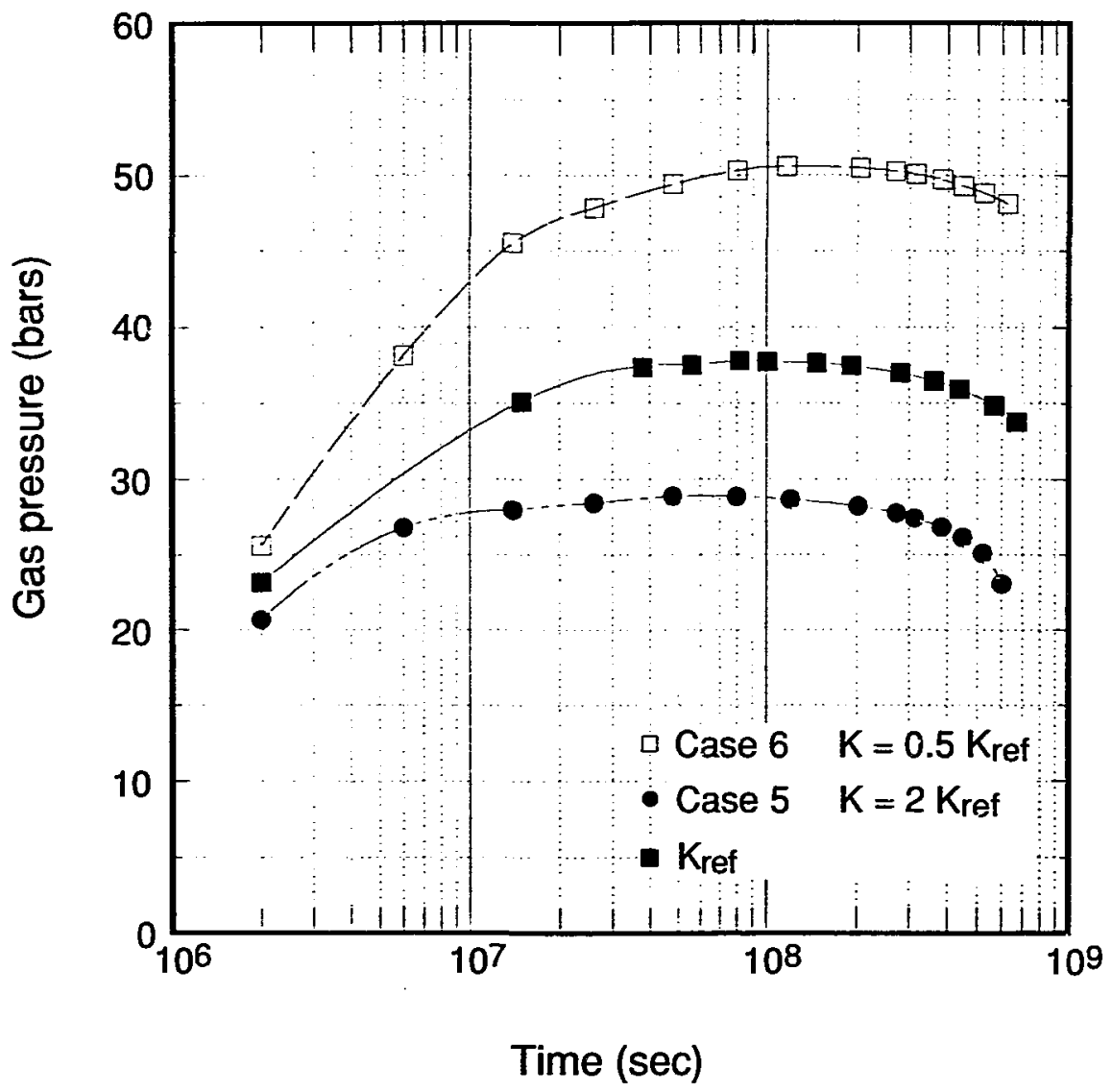

XBL $1902-6254$

Figure 19: Sensitivity of Gas Pressures in Injection Block to Variations in Absolute Permeability. 


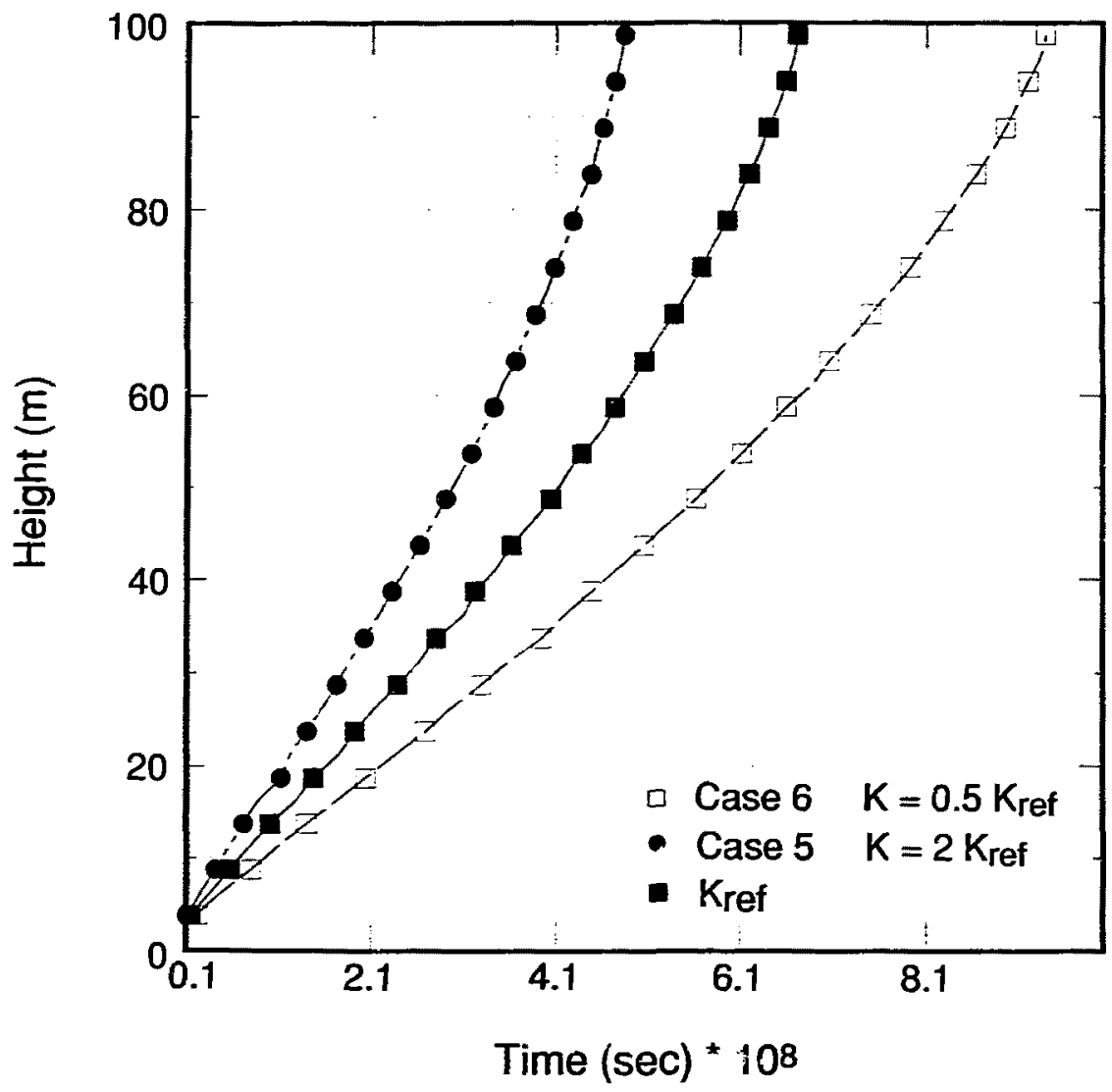

XBL 902-5255

Figure 20: Sensitivity of Gas Front Adrance to Variations in Absolute Permeability. 


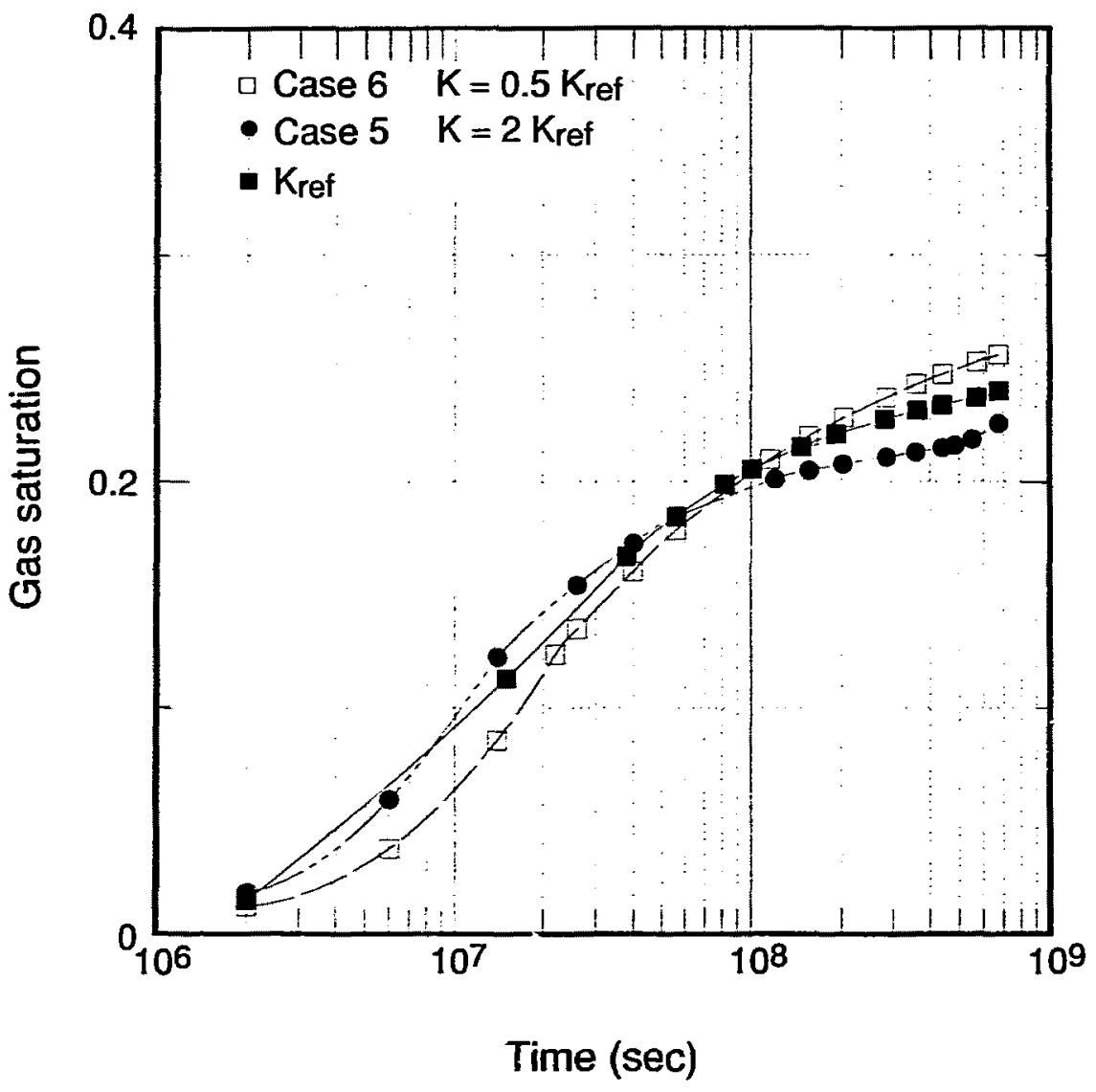

XBL 902-6256

Figure 21: Sensitivity of Gas Saturations in Injection Block to Variations in Absolute Pernteability: 


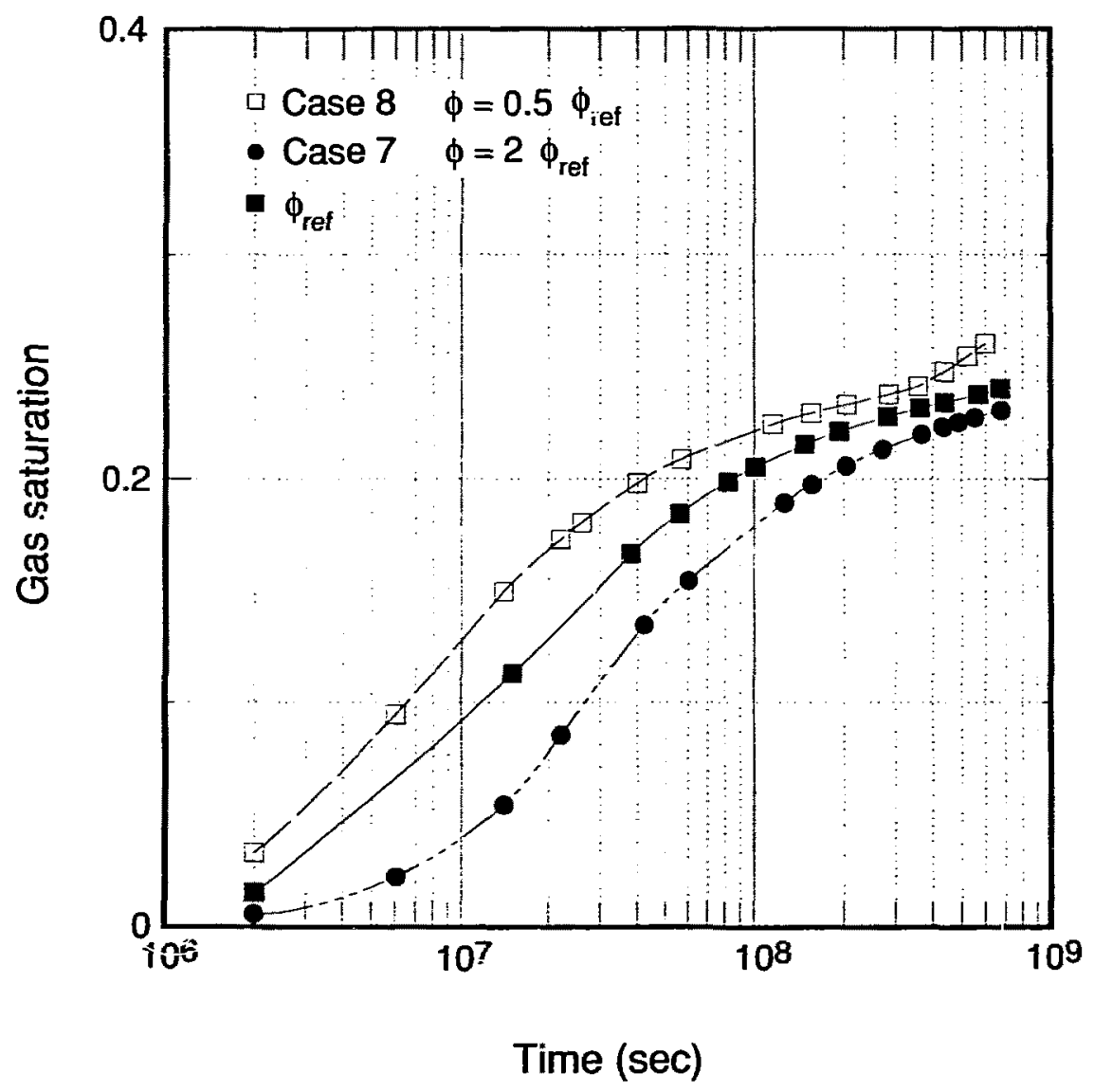

XBL 902-6257

Figure 22: Sensitivity of Gas Saturations in Injection Block to Variations in Porosity. 


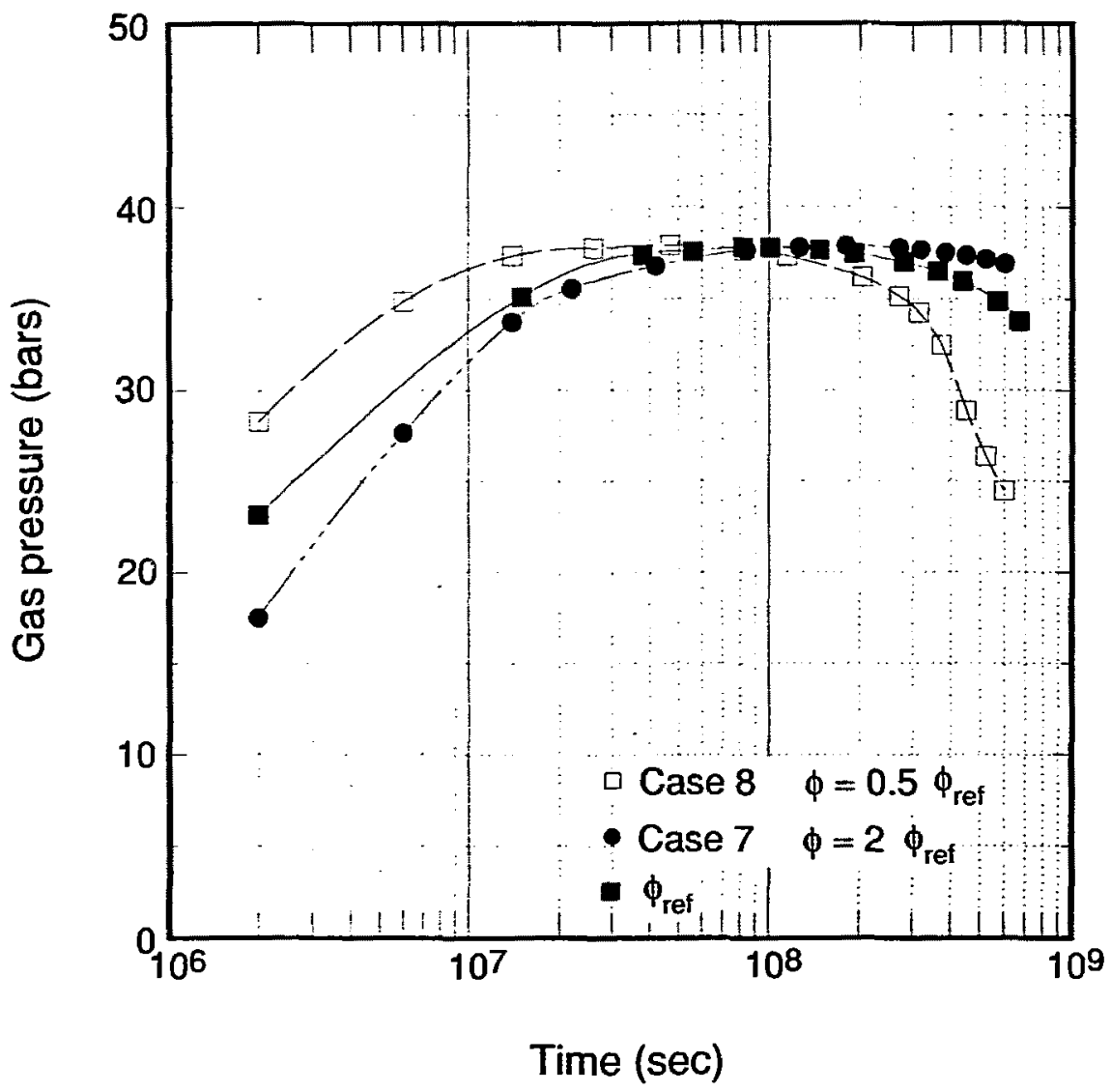

XBL 902-6258

Figure 23: Sensitivity of Gas Pressures in Injertion Block to Variations in Porosity. 


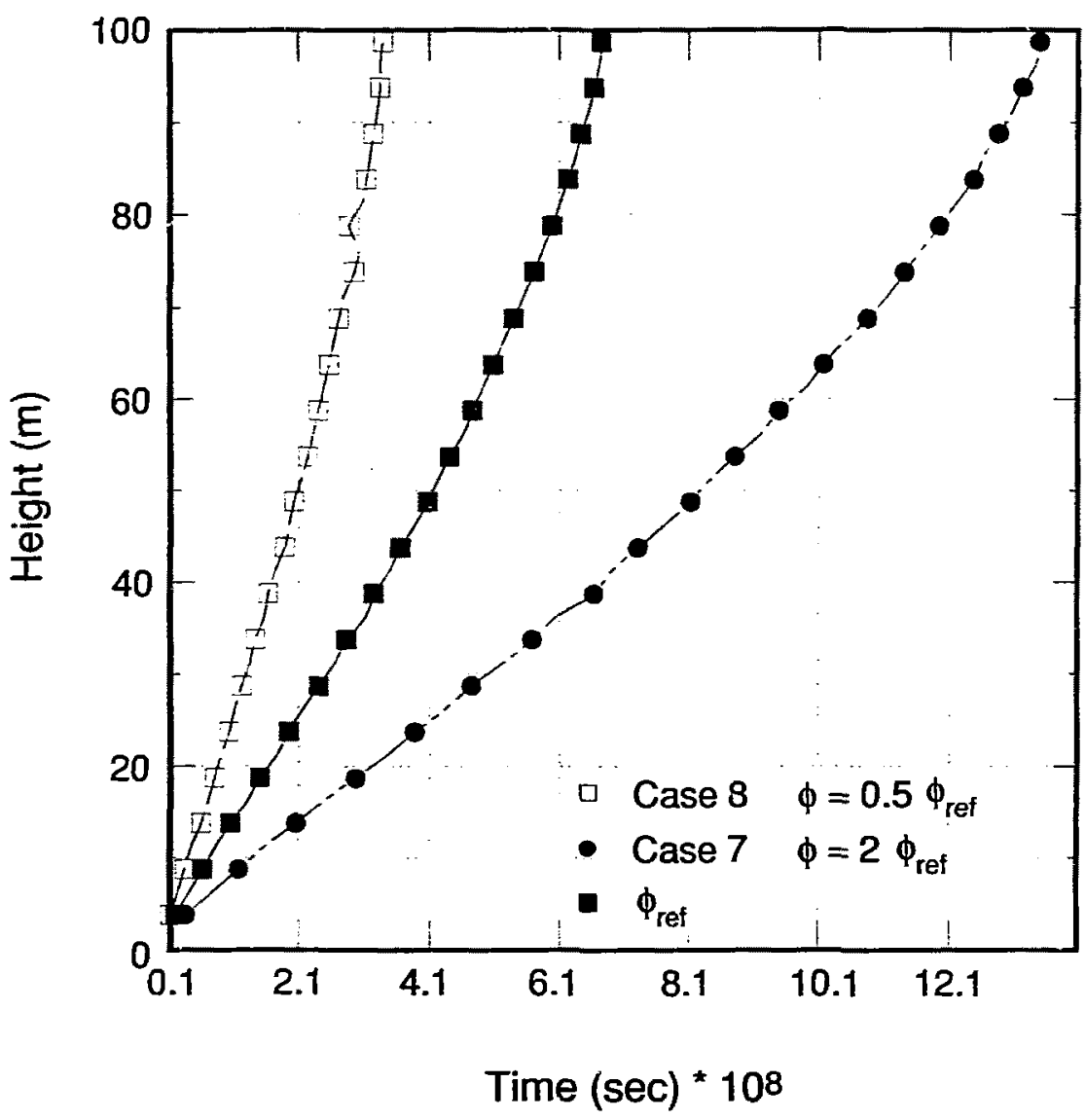

Figure 24: Sensitivity of Gas Front Advance to Variations in Porosity. XBL 902-6259 
TABLE 3. Results of Sensitivity Studies

\begin{tabular}{|c|c|c|c|}
\hline Case & $\begin{array}{c}P_{g_{\max }} \\
(\text { bars) }\end{array}$ & $\begin{array}{c}\text { time } \\
\text { (seconds) }\end{array}$ & $\begin{array}{c}\text { Gas breakthrough } \\
\text { time (seconds) }\end{array}$ \\
\hline reference & 37.98 & $0.72 \times 10^{8}$ & $0.67 \times 10^{9}$ \\
1 & 39.38 & $0.93 \times 10^{8}$ & $0.69 \times 10^{9}$ \\
2 & 37.21 & $0.72 \times 10^{8}$ & $0.66 \times 10^{9}$ \\
3 & 35.18 & $0.2 \overline{9} \times 10^{8}$ & $0.19 \times 10^{9}$ \\
4 & 93.55 & $59 \times 10^{8}$ & $3.8 \times 10^{9}$ \\
5 & 28.96 & $0.36 \times 10^{8}$ & $0.49 \times 10^{9}$ \\
6 & 50.79 & $1.00 \times 10^{8}$ & $0.94 \times 10^{9}$ \\
7 & 37.88 & $1.8 \times 10^{8}$ & $1.3 \times 10^{9}$ \\
8 & 37.95 & $0.36 \times 10^{8}$ & $0.34 \times 10^{9}$ \\
\hline
\end{tabular}

Table 3: Results of Sensitivity Studies. 


\subsection{Relative Permeability}

Interference between liquid and gaseous phases as described by relative permeability functions has an overwhelmingly strong impact on system behavior. Pressurization effects from gas release can increase dramatically when phase interference is strong, i.e., when relative permeabilities are small at intermediate saturations.

\subsection{Absolute Permeability}

- This parameter has a strong impact on pore pl..... but relatively small impact on fluid saturation.

\subsection{Porosity}

This parameter has a strong impact on the time scale of the flow processes, with things happening faster for smaller porosity. The rate of advancement of the gas front and the rate of saturation buildup are approximately inversely proportional to porosity. Pressure effects are relatively minor.

\section{CONCLUSIONS}

Major multiphase wstem parameters such as relative permeability and capillary pressure of fractured media are poorly understood at the present time. In this report we have attempted to analyze the relative impact of these and other fluid flow parameters such as porosity and intrinsic permeability on a particular two-phase system under isothermal conditions. It is important to note that due to the strong coupling effects in multiphase flow, there is a complex relationship between the input parameters and the output variables. The parameter that was shown to have by far the largest impact is relative permeability. it affects strongly both the gas pressure and the gas front displacement velocity (See Table 3). It is followed in importance by intrinsic permeability thât affects more the gas pressure values than the displacement front speed. The p:ameter that has the least impact, within the range studied here, is capillary pressure. Porosity does not have much influence on gas pressures at the injection point but it strongly affects gas saturation front velocities. 
On the other hand, intrinsic permeability has a higher impact on gas pressures than on gas front velocities.

We conclude that relative permeability constitutes a ciucial multiphase system parameter. Efforts have to be made to obtain reliable relative permeability functions that would appiy to the particular field conditions under consideration. We would like to note that the strong interference relative permeability model is expected to be more applicable to a single fracture viewed as a two-dimensional heterogeneous porous medium rather than to a three-dimensional fracture network. A three-dimensional network would present less interference between the fluid phases because it allows more alternative pathways for the phases to flow.

In our studies, we have assumed immiscible displacement of water by gas in a homogeneous sparsely fractured rock. However, the variation in apertures in any given fracture will mean that gas will tend to follow the widest aperture paths. Hence, it is conceivable that some parts of the medium may never allow gas to flow and will remain saturated with water. It is not clear a-priori to what extent the relative permeability concept being a continuum concept can be applied to flo's processes in highly heterogeneous media.

In general, we can state that our sensitivity studies are useful to:

- identify the importance (or lack thereof) of formation parameters;

- identify the most import.nt physical processes;

- quantify the extent and sources of error for prediction;

- serve as a guide for fitting a model to actual data;

- assist in the design of ficld experiments aimed at reducing uncertainty in system . parameters.

\section{ACKNOWLEDGEMENT}

This work was made possible, in part, by the U.S. Department of Energy under Contract No. DE-AC03-76SF00098, and by NAGRA, Baden, Switzerland. Thanks are due to J. 
Long, L. Myer, and L. Cox of Lawrence Berkeley Laboratory for a careful review of the manuscript. 


\section{REFERENCES}

1. Corey, A.T., 1954, The Interrelation between Gas and Oil Relative Permeabilitic:s, Producers Monthly, pp.38-41.

2. Gilman, J.R., and Kazemi, H., 1983, Improvements in Simulation of Naturally Fractured Reservoirs, Soc. Pet. Eng. J., Pp.695-707.

3. Grant, M.A., 1977, Permeability Reduction Factors at W'airakci, paper 77-HT-52,

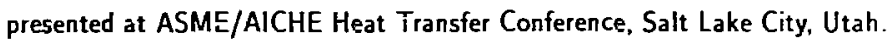

4. Narasimhan, T.N., Witherspoon, P.A., and Edwards, A.L., 1978, Numerical Model for Saturated-linsaturated Flow in Deformable Porous Media, Part 2: The A/gorithm, Water Resources Research, Vol.14, No. 2, pp.255-261.

5. Pruess, K., 1987, TOUGH User's Guide. Nuclear Regulatory Commission, Report NUREG/CR-4645 (also: Lawrence Berkeley Laboratory Report LBL-20700, Berkeley. Ca.).

6. Pruess, K., 1989. Numerical Modelling of Gas .1Figration at a Proposed Repository for Low and Intrnediate Level Nuclear Wastes at Oberbuenstock, Suitzevitanl, Lawrence Berkeley Report LBL-25413, NDC-5.

7. Pruess, K., and Tsang, Y.W., 1989, On Relative Permeability of Rough-Walled Fractures, Lawrence Berkeley Laboratory Report LBL-26509, Berkeley, Ca. Report LBL-20700, Berkeley, Ca.

8. Pruess, K., 1990, TOUGH2-A General-Purpose Simulator for Mtultiphase Nonisothermal Flows, draft manuscript.

9. Schlueter, E., 1989, On Relative Permeability and Capillary Pressure of Gas and Water in Porous and Fractured Media, Mineral Engineering 290A-Course Report, U.C. Berkeley, Ca.

10. Wiborgh, M., Hoglund, L.O., and Pers, K., 1986. Gas Formation and Gas Transport in the Host Rock, NAGRA Report NTB 85-17. 


\section{APPENDIX A. GAS RELEASE RATES}

The gas release rate was obtained as follows:

Top surface area of repository is $700 \times 1=700 \mathrm{~m}^{2}$

$Q_{g}=3.3 \times 10^{-\pi} \mathrm{kg} / \mathrm{s}$ of hydrogen over $700 \mathrm{~m}^{2}$ (Pruess. 1989).

Equivalent $Q_{g}$ for $1 \mathrm{~m}^{2}=3.3 \times 10^{-i} / 700=4.7 \times 10^{-10} \mathrm{~kg} / \mathrm{s}$

To scale for air (same rate on molar or volumetric basis):

From ideal gas law,

$P_{g} V_{g}=(m / M) R T$

where $\quad M_{H_{2}}=\mathbf{2 . 0 1 6}$

$$
M_{\text {air }}=28.96
$$

Thus,

$Q_{g, e q, a i r}=(28.96 / 2.016) \times 4.7 \times 10^{-10}=6.75 \times 10^{-9} \mathrm{~kg} / \mathrm{s} \times \mathrm{m}^{2}$

The rate used was: $Q_{g}=10^{-8} \mathrm{~kg} / \mathrm{s} \times \mathrm{m}^{2}$

\section{APPENDIX B. CAPILLARY PRESSURE FOR A FRAC- TURE WITH LOG-NORMAL APERTURE DISTRI- BUTION}

\section{B.1 Function Origin}

Laboratory measurements of fracture void space geometry have shown that, in many cases, apertures follow a log-normal distribution. Under those conditions, a relationship between liquid saturation and aperture can be obtzined in closed form, as follows (Pruess and Tsang. 1989)

$$
S_{l}=1 / 2\left(1+\operatorname{erf}\left[\left(\log \left(b_{p}\right)-\beta\right) / \sqrt{\left.2 \sigma^{2}\right]}\right) ; \log \left(b_{p}\right)-\beta>0\right.
$$




$$
S_{l}=1 / 2\left(1-\operatorname{\epsilon rf}\left[\left(\log \left(b_{p}\right)-\beta\right) / \sqrt{\left.2 \sigma^{2}\right]}\right) ; \log \left(b_{p}\right)-\beta<0\right.
$$

where $\quad S_{l} \equiv$ liquid saturation

$$
\begin{aligned}
& b_{p} \equiv \text { cut-off aperture (apertures } b<b_{p} \text { containing liquid, } b>b_{p} \text { gas) } \\
& \sigma \equiv \text { variance } \\
& \bar{b} \equiv \text { mean aperture } \\
& \beta=\sigma^{2} \ln (10)+\log \left(\bar{b} / \epsilon^{(\sigma \ln (10))^{2} / 2}\right)
\end{aligned}
$$

Note that $\beta$ is the logarithm of the aperture cutoff corresponding to $S_{l}=50 \%$.

Eqs.(1,2) can be rewritten as follows,

$$
S_{t}=1 / 2\left(1+\operatorname{cr} f\left[\left(\log \left(b_{p}\right)-\beta\right) / \sqrt{2 \sigma^{2}}\right]\right)
$$

Thus,

$$
b_{p}=10^{3-\sqrt{2} \sigma e r f i\left(1-2 S_{l}\right)}
$$

where $\operatorname{\epsilon r} f i \equiv$ inverse error function

The capillary pressure is defined by Laplace's equation. Assuming a contact angle of zero, we have

$$
P_{c}=2 \gamma / b_{p}=2 \gamma 10^{\sqrt{2} \sigma \operatorname{er} f i\left(1-2 S_{l}\right)-\beta}
$$

where $\gamma \equiv$ air-water surface tension $(\approx 0.073 \mathrm{~N} / \mathrm{m})$

\section{B.2 Parameter Adjustment}

The theoretically-based capillary pressure curve for $\sigma=0.43$ and $\bar{b}=81.8 \mu \mathrm{m}$ as used by Pruess and Tsang (1989) is presented in Fig.25. This curve was fitted with the 'limestone' curve of Wiborgh et al. (1986) at $S_{l}=50 \%$ as follows: (Fig.26)

$$
P_{c}=3.15 \times 10^{5} \mathrm{~N} / \mathrm{m}^{2} \text { at } S_{t}=50 \% \text { for "limestone". }
$$

\section{Thus.}




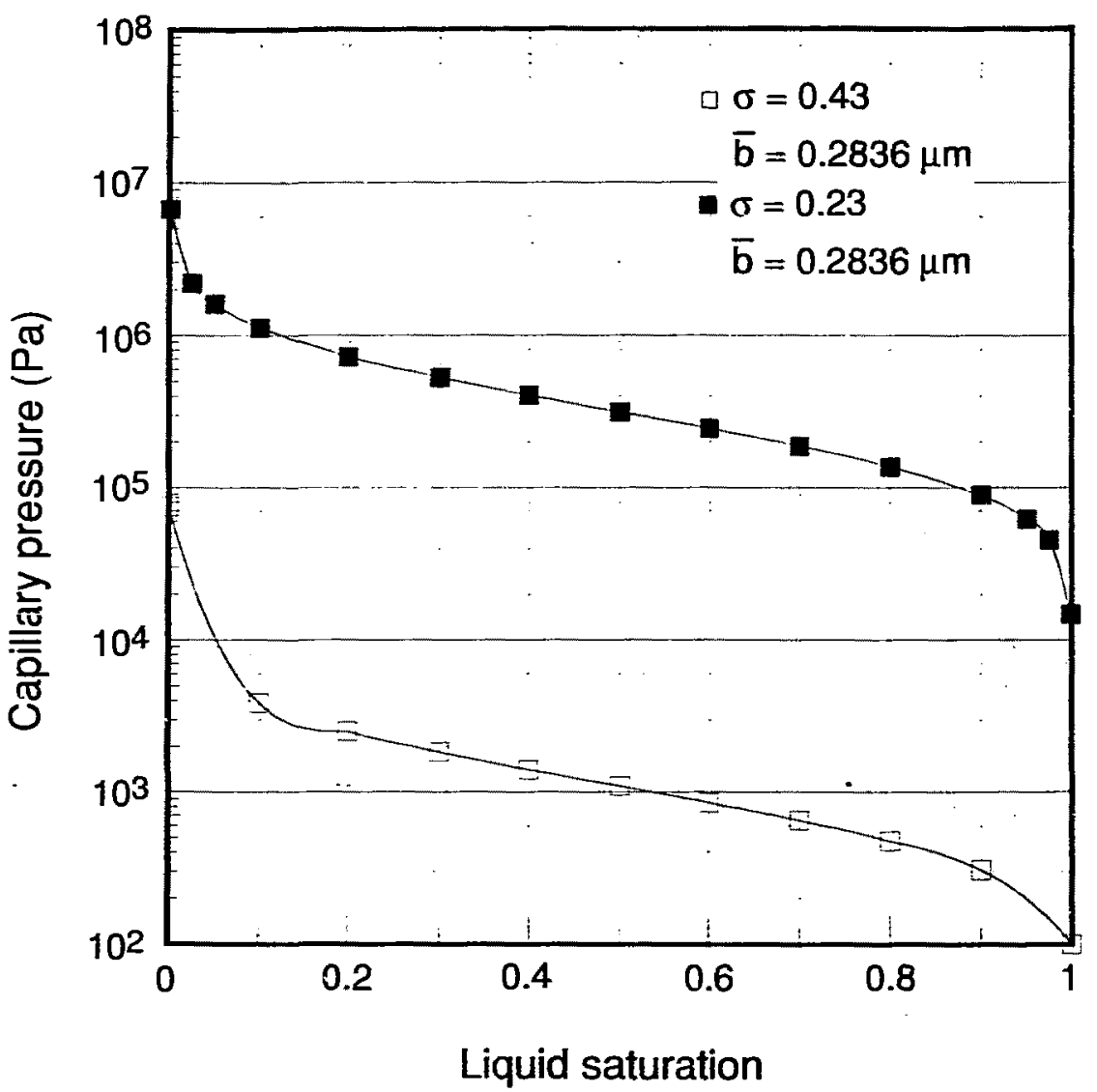

Figure 25: Fracture Capillary Pressures for Log-Normal Aperture Distribution.

XBL 902-6260 


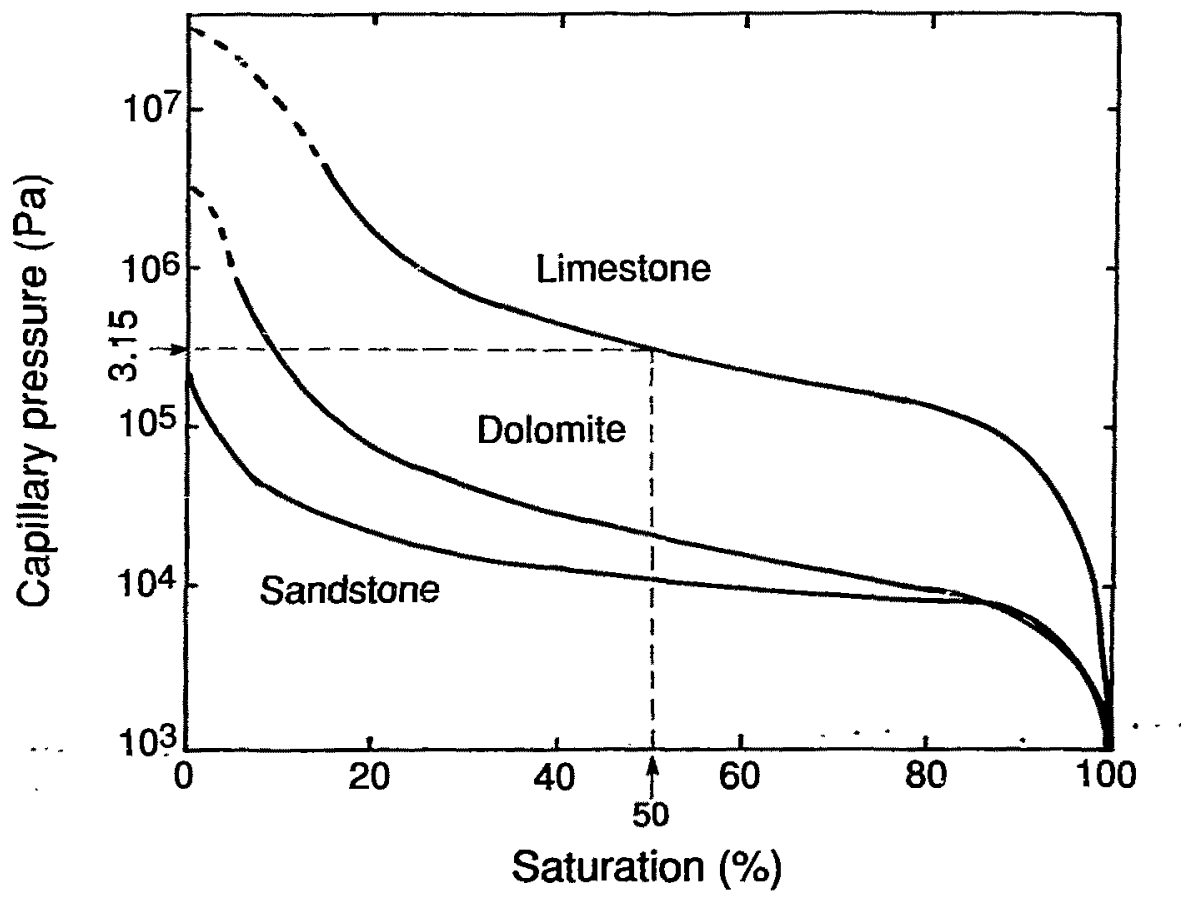

XBL 902-6261

Figure 26: Capillary Pressure Functions for Three Comun Geologic Media (From Wiborgh et . al., $1985)$. 
$b_{p_{e q}}=2 \gamma / P_{c}=(2 \times 0.073 \mathrm{~N} / \mathrm{m}) /\left(3.15 \times 10^{5} \mathrm{~N} / \mathrm{m}^{2}\right)=0.0163 \times 10^{-5} \mathrm{~m}=0.16 .3 \mathrm{Hm}$

Therefore,

$\beta=\log \left(b_{p_{\mathrm{eq}}}\right)=\log (0.463)=-0.3344$

From Eq.(3). (with $\sigma=\mathbf{0 . 4 3 )}$

$\beta=-0.3344=(0.43)^{2} \ln (10)+\log \left(\bar{b} / e^{(0.43 \ln 10)^{2} / 2}\right)$

Hence,

$\bar{b} \approx 0 . .836 \mu m$

Thus the parameters of the capillary pressure for the reference case are: (Fig.25)

$\sigma=0.43, \bar{b}=0.2 S 36, \mu m, \beta=-0.3344$

\section{B.3 Numerical Implementation}

For use in the TOUGH simulator, capillary pressure must be calculated as a function of saturation. This involves inversion of the relationships Eqs. $(1,2)$ or $(3)$.

The programming was executed in two alternative ways:

1. Using eqs.(1,2) (See Code Listing 1)

2. Using eq. (3) (See Code Listing 2)

\section{B.3.1 Inverse Error Function}

As it is presented in Eqs.(4.5), the expression that relates aperture size or capillary pressure with liquid saturation contains the inverse error function (Fig.27). The error function analytical inyersion is not a trivial operation. A Cray - IMSLSFUN mathematical library function (erfi(x)), was implemented in TOLGH2 to calculate the inverse error function.

Restrictions of erf( $(x)$ :

$-1<x<1$ 


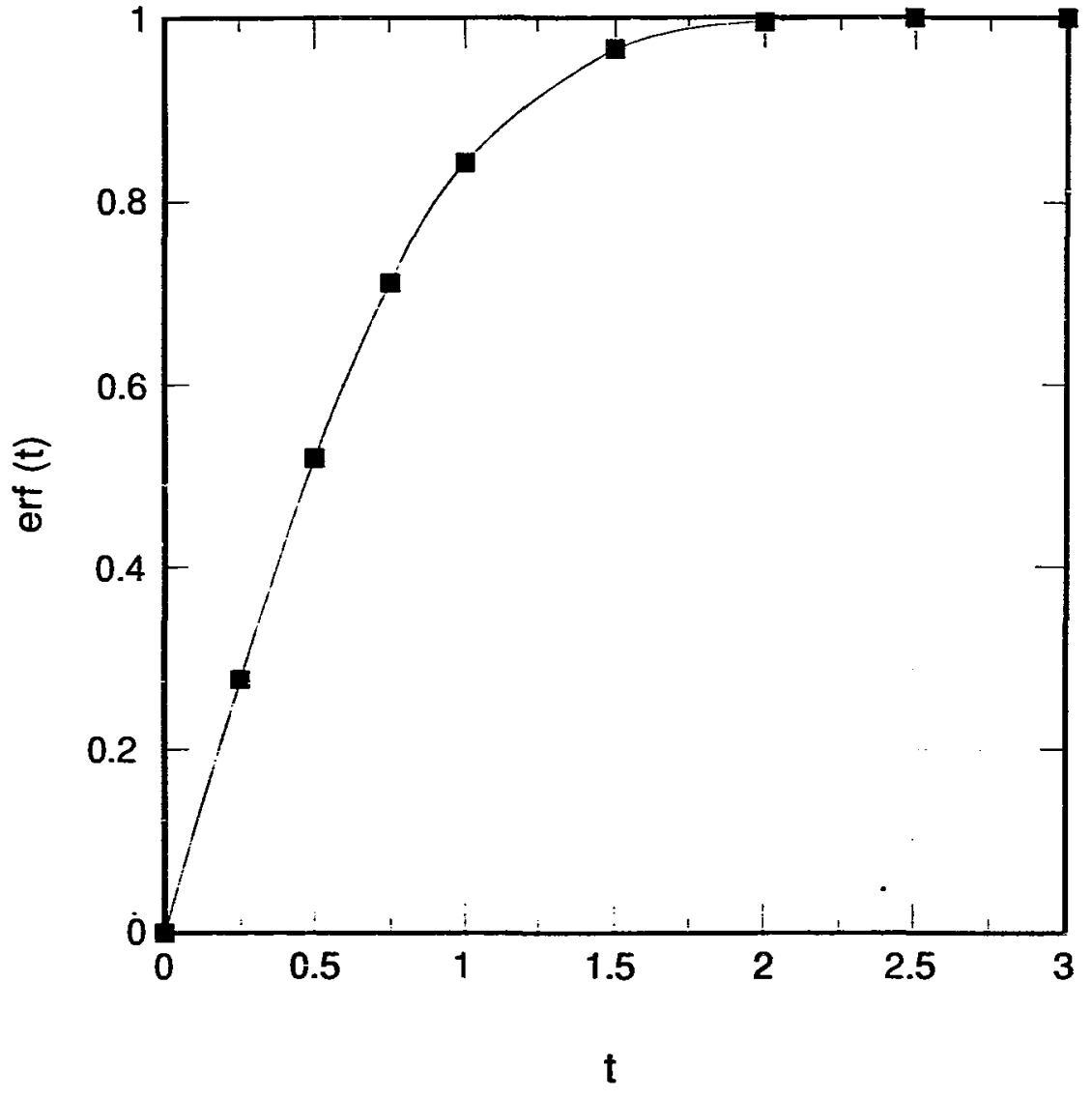

XBL 902-6262

Figure 2:: The Error Function 
- data may be real or double precision if the double precision version derfi( $x$ ) is used. Results of derfi(x) is accurate to less than one half precision if the absolute value of the argument is too large (Terrie Dickson (LLNL), private cc. imunication).

\section{B.3.2 Access Procedure of Erfi(x) on the Cray (See Code Listing 3)}

\section{B.3.3 Treatment of Singularities}

As it is shown in Fig.27, erfi $(+1)$ is not defined. Also, erfi(-1) =-erfi(1) is not defined either. From formula (5), it is obvious that at values of saturation $S_{l}=0$ and $S_{l}=1$; $\operatorname{tr} f i(x)=\operatorname{er} f i(+1)$ and $\operatorname{er} \int i(x)=\operatorname{er} \int i(-1)$ respectively. Therefore, a special treatment is required for the mathematical singularities at $S_{l}=0,1$ (See Fig.2).

Study first how the function $P_{c}$ vs. $S_{l}$ behaves for:

- Increasingly small $S_{l}$ values: (e.g. $S_{l} \rightarrow 0$, Table $4 a$ )

- Increasingly large $S_{l}$ values: (e.g. $S_{I} \rightarrow 1$, Table $4 b$ )

From the observed behavior $\left(S_{l} \rightarrow 0, P_{c} \rightarrow \infty ; S_{l} \rightarrow 1, P_{c} \rightarrow 0\right)$, the following solution was proposed. For small liquid saturation, we impose a cutoff

- For $S_{l} \leq 10^{-5}: P_{c}=P_{c_{b}}$, where $P_{c_{b}} \equiv P_{c}$ at $S_{l}=10^{-5}$; while for large liquid saturation we perform a linear interpolation.

- For $S_{l} \geq 1-10^{-5}: P_{c}=P_{c_{t}}\left(1-S_{l}\right) / 10^{-5}$, where $P_{c_{\mathrm{t}}} \equiv P_{c}$ at $S_{l}=1-10^{-5}$. (Note that at $S_{l}=1, P_{c}=0$ ).

\section{B.3.4 Test Cases}

Test cases were performed to study:

1. Erfi(x) performance.

2. Comparison of analytical solution using the probability table with the numerical solution proposed (Table 5). (Pick cut-off aperture $b_{p}$ and calculate liquid saturation $S_{l}$ and capillary pressure $P_{\text {qanal }}$. Then, use the code with $S_{l}$ to calculate $P_{c[r i m !}$ and compare the two capillary pressure values thus obtained). 
TABLE 4a. Behavior of fracture capillary pressure for $S_{l} \rightarrow 0$

\begin{tabular}{|c|c|}
\hline$S_{l}$ & $P_{c}$ \\
& $(P . A)$ \\
\hline $10^{-4}$ & $-0.1253 \times 10^{8}$ \\
$10^{-5}$ & $-0.21 .51 \times 10^{8}$ \\
$10^{-6}$ & $-0.3459 \times 10^{8}$ \\
\hline
\end{tabular}

TABLE 4b. Behavior of fracture capillary pressure for $S_{l} \rightarrow 1$

\begin{tabular}{|c|c|}
\hline$S_{l}$ & $\begin{array}{c}P_{c} \\
(P A)\end{array}$ \\
\hline $1-10^{-4}$ & $-0.7926 \times 10^{4}$ \\
$1-10^{-5}$ & $-0.4622 \times 10^{4}$ \\
$1-10^{-6}$ & $-0.2850 \times 10^{4}$ \\
\hline
\end{tabular}

Table 4: Behavior of Fracture Capillary Pressure for (a) $S_{l}-0$. (b) $S_{l}-1$. 
TABLE 5. Numerical Check of Fracture Capillary Pressure Function

\begin{tabular}{|c|c|c|c|c|c|}
\hline $\begin{array}{c}b_{p} \\
(\mu m)\end{array}$ & $\left(\log \left(b_{p}\right)-\beta\right) / \sigma \sqrt{2}$ & $\epsilon r f\left(\log \left(b_{p}\right)-\beta / \sigma \sqrt{2}\right)$ & $S_{t}\left(b_{p}\right)$ & $\begin{array}{c}P_{\text {Ianal }_{\text {Ia }}}\left(b_{p}\right) \\
(\mathrm{Pa})\end{array}$ & $P_{(\mathrm{Pa})}\left(b_{p}\right)$ \\
\hline .05 & -1.5902 & .97546 & .0123 & $-2.9 \times 10^{6}$ & $-2.945 \times 10^{6}$ \\
\hline .1 & -1.0952 & .87851 & .06 .07 & $-1.5 \times 10^{6}$ & $-1.457 \times 10^{6}$ \\
\hline .2 & -.6002 & .60543 & .197 & $-7.3 \times 10^{5}$ & $-7.328 \times 10^{5}$ \\
\hline .332 & -.2382 & $.2635 \tau$ & .367 & $-4.4 \times 10^{5}$ & $-4.411 \times 10^{5}$ \\
\hline .6 & .1844 & .20530 & .592 & $-2.4 \times 10^{5}$ & $-2.503 \times 10^{5}$ \\
\hline .8 & .3899 & .41874 & .695 & $-1.8 \times 10^{5}$ & $-1.902 \times 10^{5}$ \\
\hline 1.0 & .5492 & .56249 & .781 & $-1.5 \times 10^{5}$ & $-1.462 \times 10^{5}$ \\
\hline 2.0 & 1.044 & .86017 & .930 & $-7.3 \times 10^{4}$ & $-7.309 \times 10^{9}$ \\
\hline 3.0 & 1.334 & .94097 & .971 & $-4.9 \times 10^{4}$ & $-4.095 \times 10^{1}$ \\
\hline
\end{tabular}

Table 5: Numerical Check of Fracture Capillary Pressure Function $(\beta=-.3341 ; \sigma=43 ; \bar{b}=$ $.2838 \mu m)$. 
3. Handling at the boundaries $S_{l} \rightarrow 0, S_{l} \rightarrow 1$.

4. Comparison of codings using Eqs.(1,2) and Eq.(3) respectively.

5. Comments:

- noted that erfi $(x)$ is very accurate.

- the analytical solution checks against the numerical solution.

- the boundaries behave well numerically.

- codings using Eqs.(1,2) and Eq.(3) are both currect.

B.3.5 $P_{e}$ vs. $S_{l}$ Functions for Differtnt $\sigma, \bar{b}$

- Constant $\sigma=0.43$ (Fig.25)

$\overline{b_{1}}=81.8 \mu \mathrm{m}$

$\overline{b_{2}}=0.2836 \mu m$

Note that the curve simply translates vertically parallel to the capillary pressure axis since the range of apertures is scaiea by a common factor, e.g. $P_{c} \approx 1 / b$. The thcoretically-based capillary pressure-saturation relation depends only on the variance of the lognormal distribution and the ratio of tive most probable aperture to the cutoff aperture. Therefore, the relationship between capiliary pressure and saturation for fractures with lognormal aperture distribution for fixed standard deviation has tio same functional form independently of the magnitude of the most probable aperture. If all apertures are scaled by a common factor, the capillary pressure at a given saturation will scale by the inverse of that factor.

- Constant $\bar{b}=0.2836 \mu \mathrm{m}$ (Fig.28)

$$
\begin{aligned}
& \sigma_{1}=0.23 \\
& \sigma_{2}=0.43 \\
& \sigma_{3}=0.63
\end{aligned}
$$

Note that there is leftward rotation and flattening of the curves with decreasing variance. 


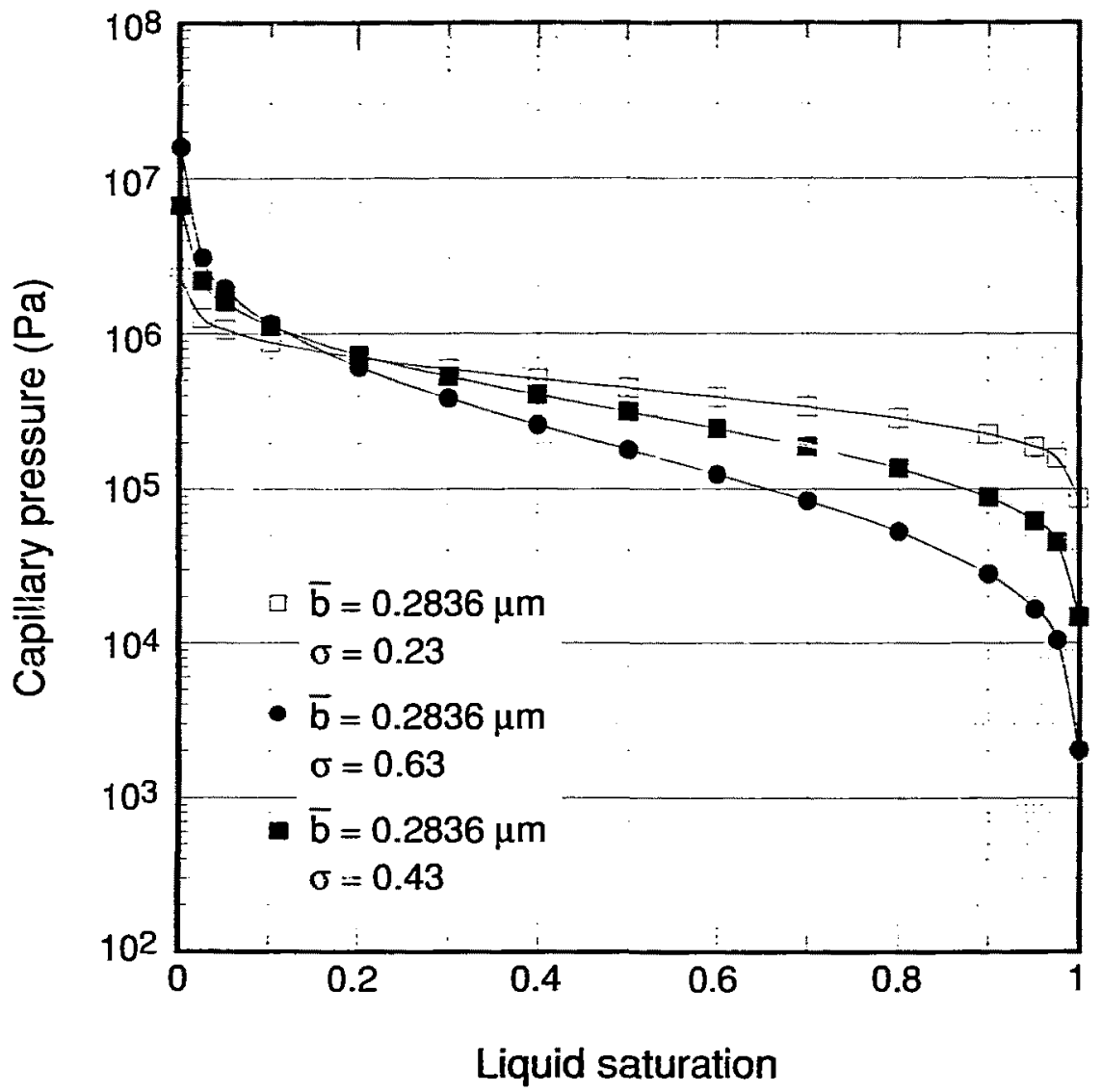

XBL 902-6263

Figure 28: Fracture Capillary Pressures for Different Values of Variance $\sigma$. 


\section{APPENDIX C. STRONG INTERFERENCE RELATIVE PERMEABILITY FUNCTIONS}

The strong interference relative permeability functions used in this studies were described by the following polynomials:

$$
\begin{aligned}
& S_{1}^{*}=\left(S_{l}-S_{l_{r}}\right) /\left(1-S_{l_{r}}\right) \\
& S_{2}^{*}=\left(S_{g}-S_{s_{r}}\right) /\left(1-S_{s_{r}}\right) \\
& k_{r_{l}}=\left(S_{1}^{*}\right)^{4} \\
& k_{r_{g}}=\left(S_{2}^{*}\right)^{4}
\end{aligned}
$$

These functions as coded in TOUGH2 are presented in Listing 5 and plotted in Fig.15. 


\section{CODE LISTINGS}

D.1 FORTRAN Source Code for Calculating Fracture Capillary Pressure (Version 1).

D.2 FORTRAN Source Code for Calculating Fracture Capillary Pressure (Version 2).

D.3 Access Procedure for Inverse Error Function from $I M S L S F T^{\circ} \mathrm{Li}$ brary.

D.4 Coding for Identifying the Position of the Gas Saturation Front.

D.5 Coding for Relative Peimeability Function with Strong Phase Interference.

D.6 Sample TOL:GII2-Input File for Reference Case. 


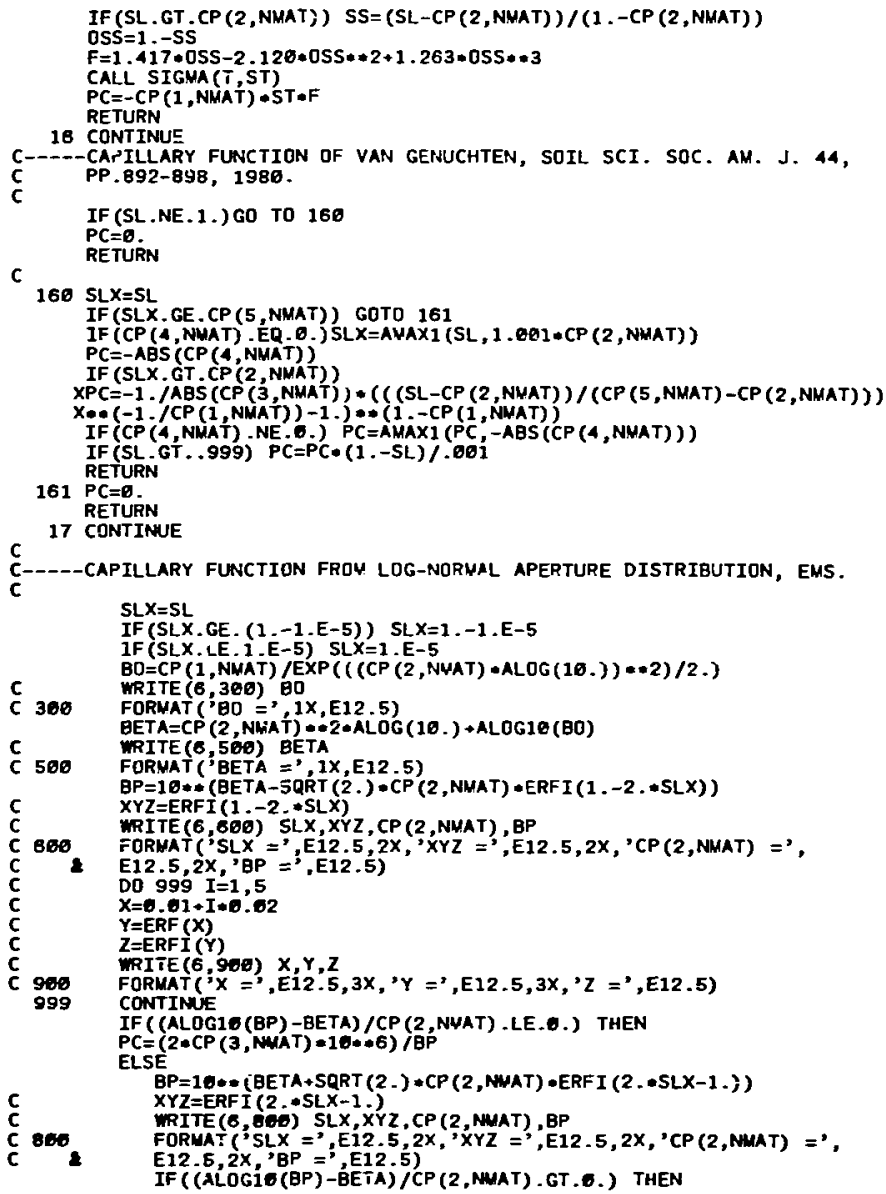

D.1 FORTRAN Source Code for Calculating Fracture Capillary Pressure (Version 1). 


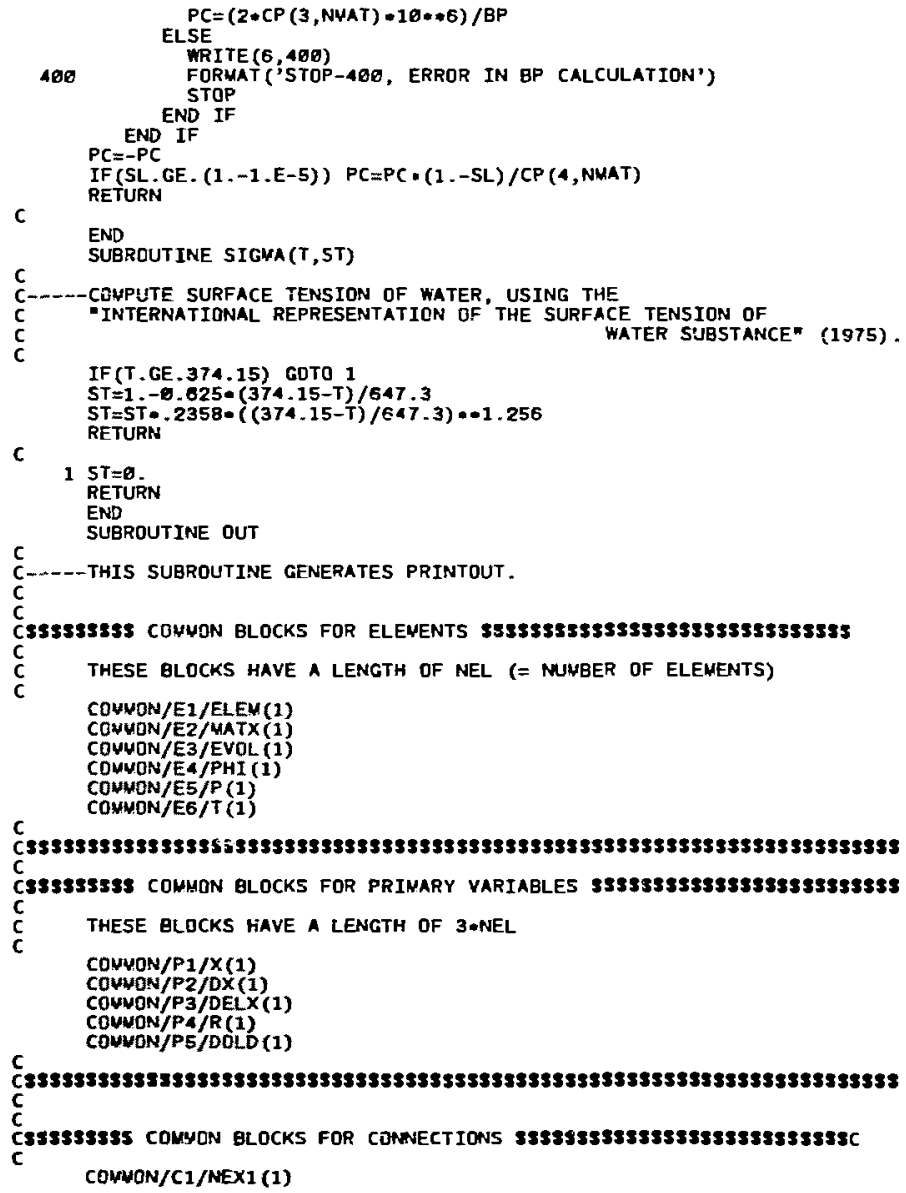

$15 T=0$

RETURN

END

SUBROUTINE OUT

C

c

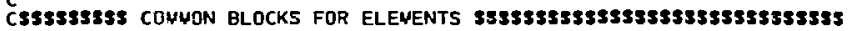

c THESE bloCKS HAVE ^ LENGTH OF NEL (= MUMBER OF ELEMENTS)

COUWON/E1/ELEN(1)

CONUON/EZ/MATX(1)

CQUMON/E3/EVOL (1)

CONUON/EA/PHI (1)

CONWON/ES/P (1)

CONNON/EG/T (1)

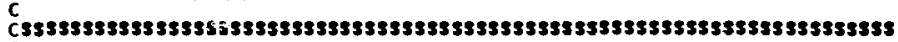
C c THESE BLOCKS HAVE A LENGTH OF 3.NEL

CONYON/P1/X(1)

COUVON/P2/DX(1)

COUNON/P3/DELX(1)

CONNON/P4/R (1)

C COUVON/P5/DOLD (1)

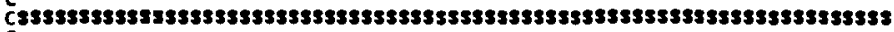

c Csssssssss COMYON GLOCKS FOR CONWECT IONS ssssssssss3sss3ssssssssssseC c

COWNON/C1/NEX1 (1) 


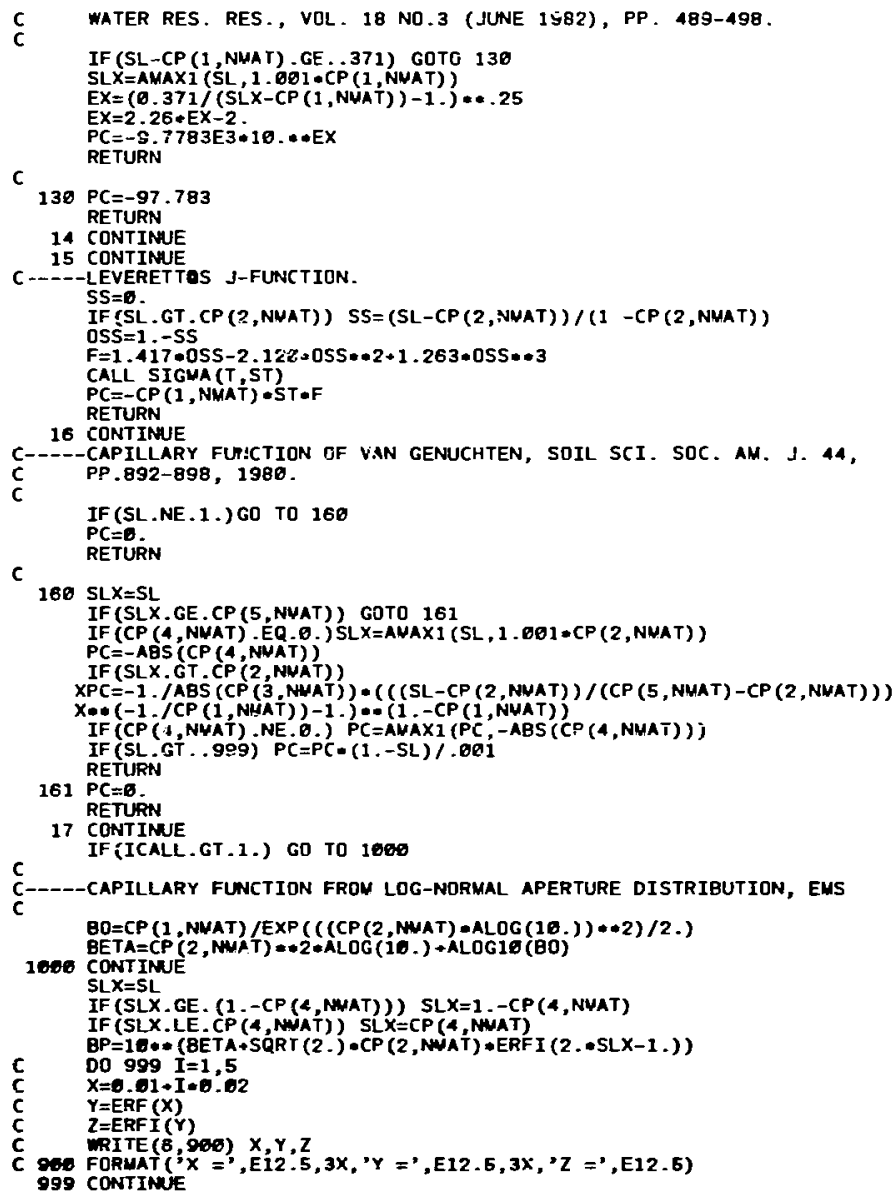

D.2 FORTRAN Source Code for Calculating Fracture Capillary Pressure (Version 2). 


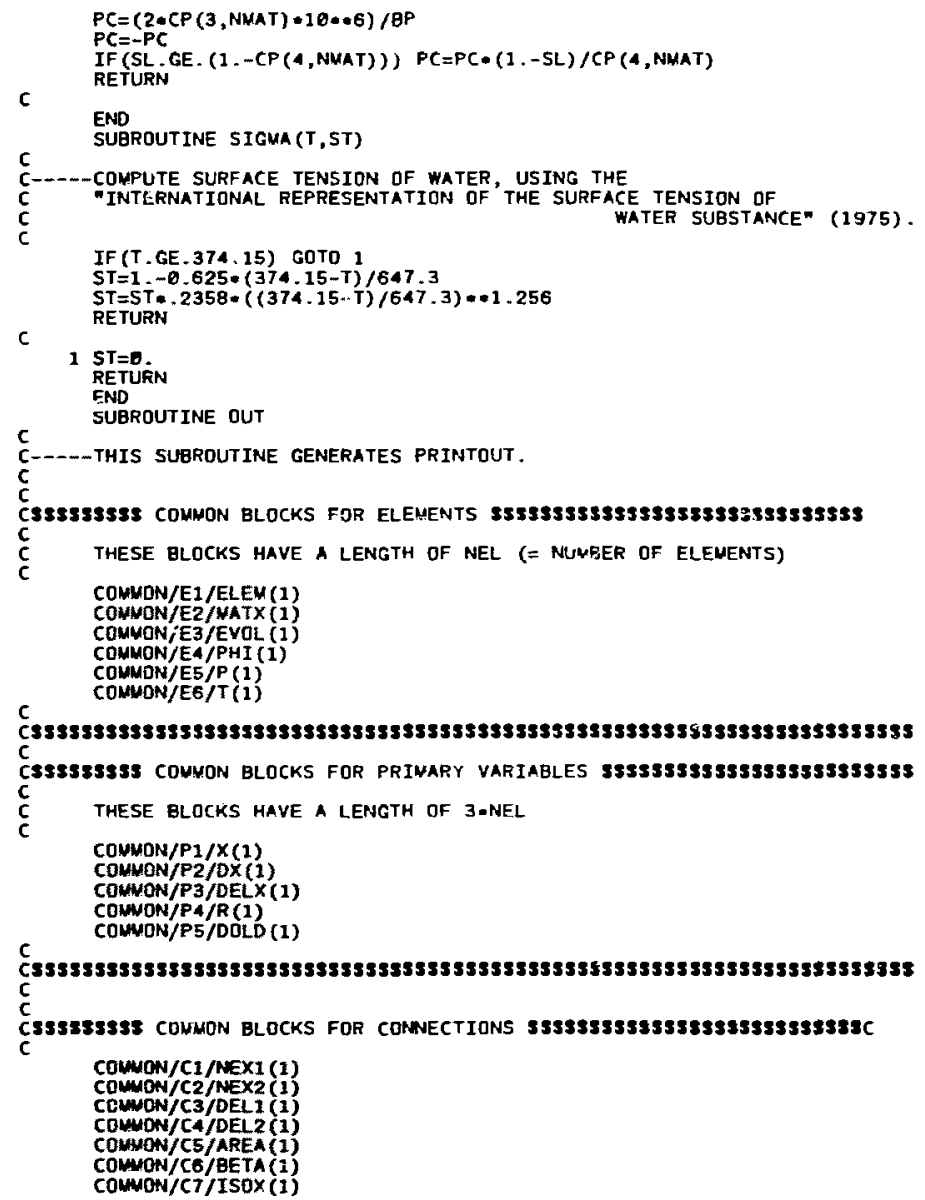




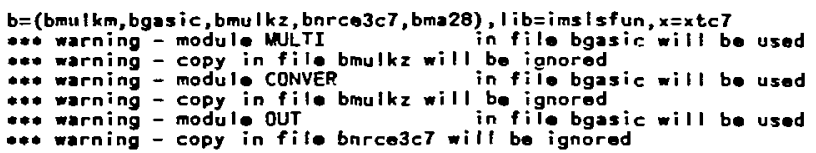

following is a list of relocatable binaries specified for this load. they will be referred to by their ordinal elsewhere in this load map.

ordinal

name

$\begin{aligned} 1 & \text { bmulkm } \\ 2 & \text { bgasic } \\ 3 & \text { bmulkz } \\ 4 & \text { bnrce3c7 } \\ 5 & \text { bma28 } \\ 6 & \text { imslsfun } \\ 7 & \text { imslcor } \\ 8 & \text { fortlib } \\ 9 & \text { mothlib } \\ 16 & \text { omnilib } \\ 11 & \text { stacklib } \\ 12 & \text { baselib }\end{aligned}$

libraries used

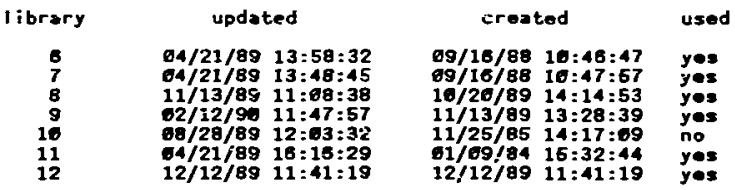

\section{D.2 Access Procedure for Inverse Error Function from IMSLSFUN Li- brary.}




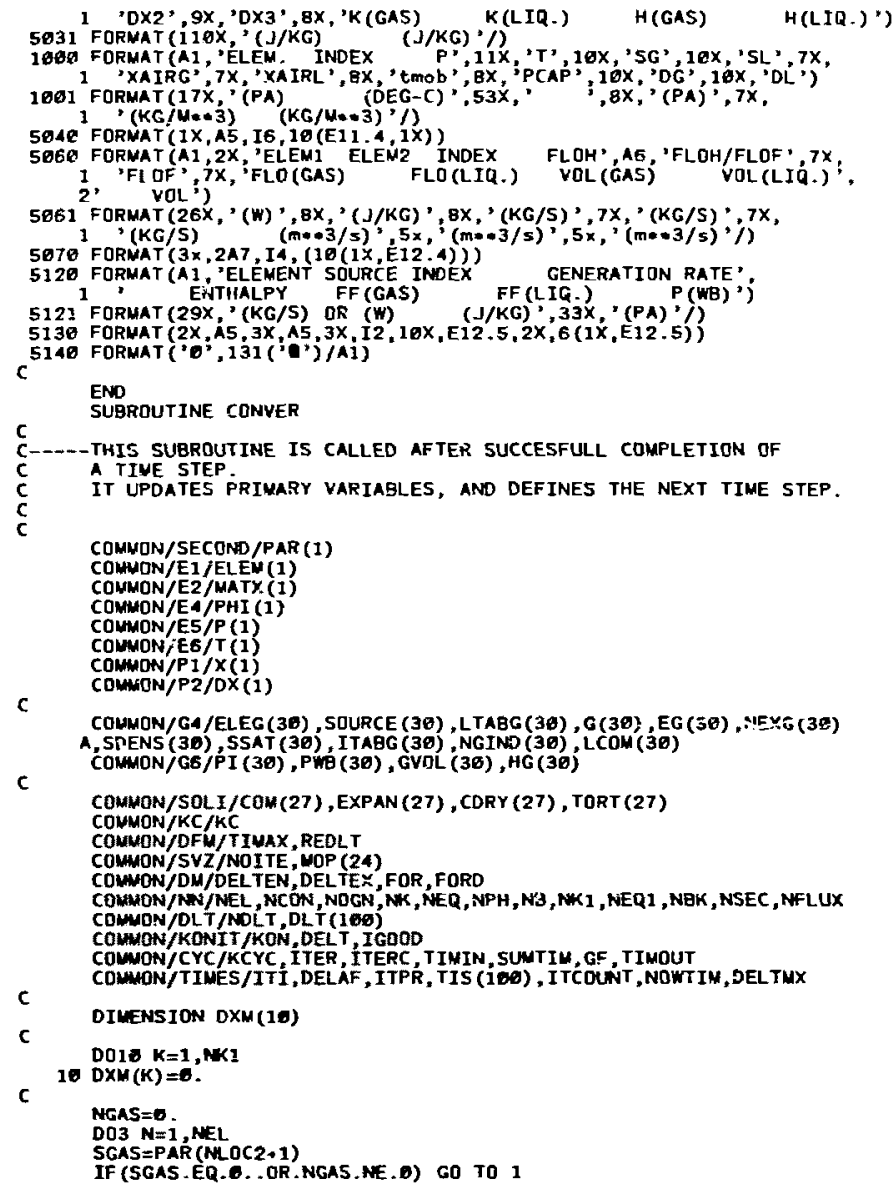

\section{D.4 Coding for Identifying the Position of the Gas Saturation Front.}




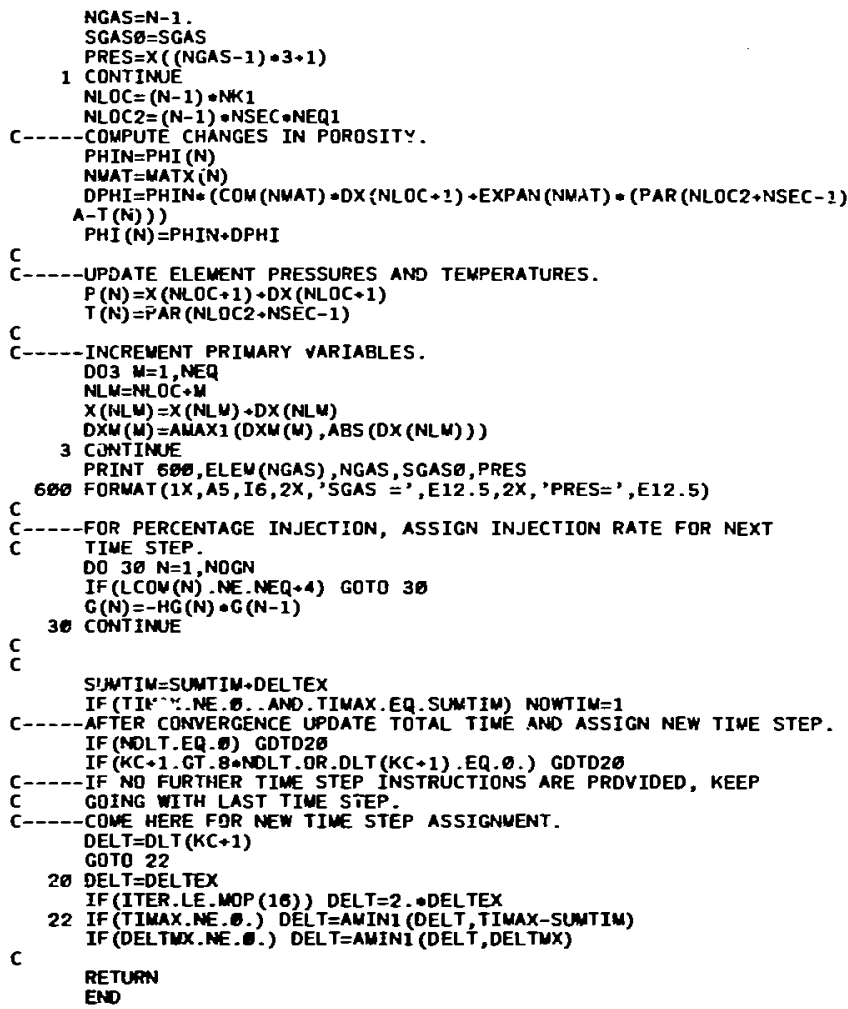




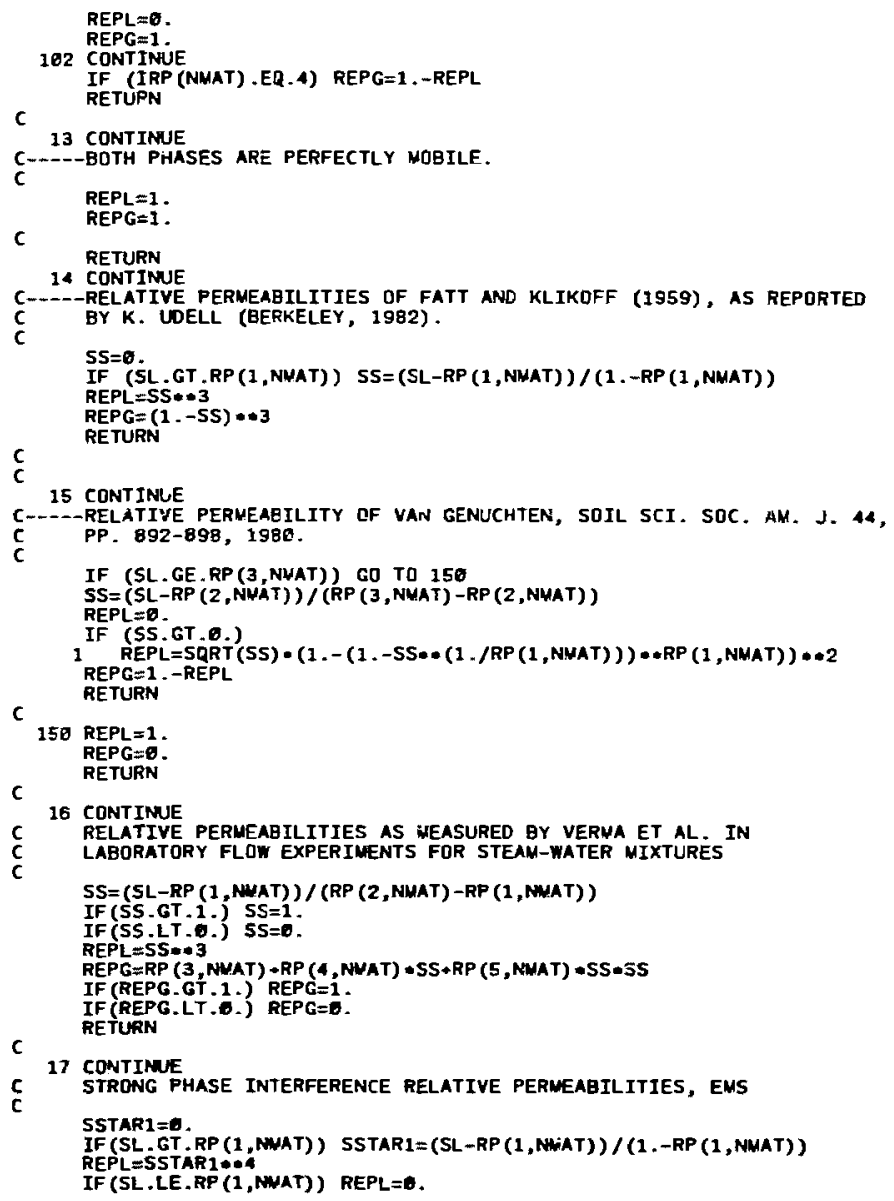

D.5 Coding for Relative Permeability Function with Strong Phase Interference. 


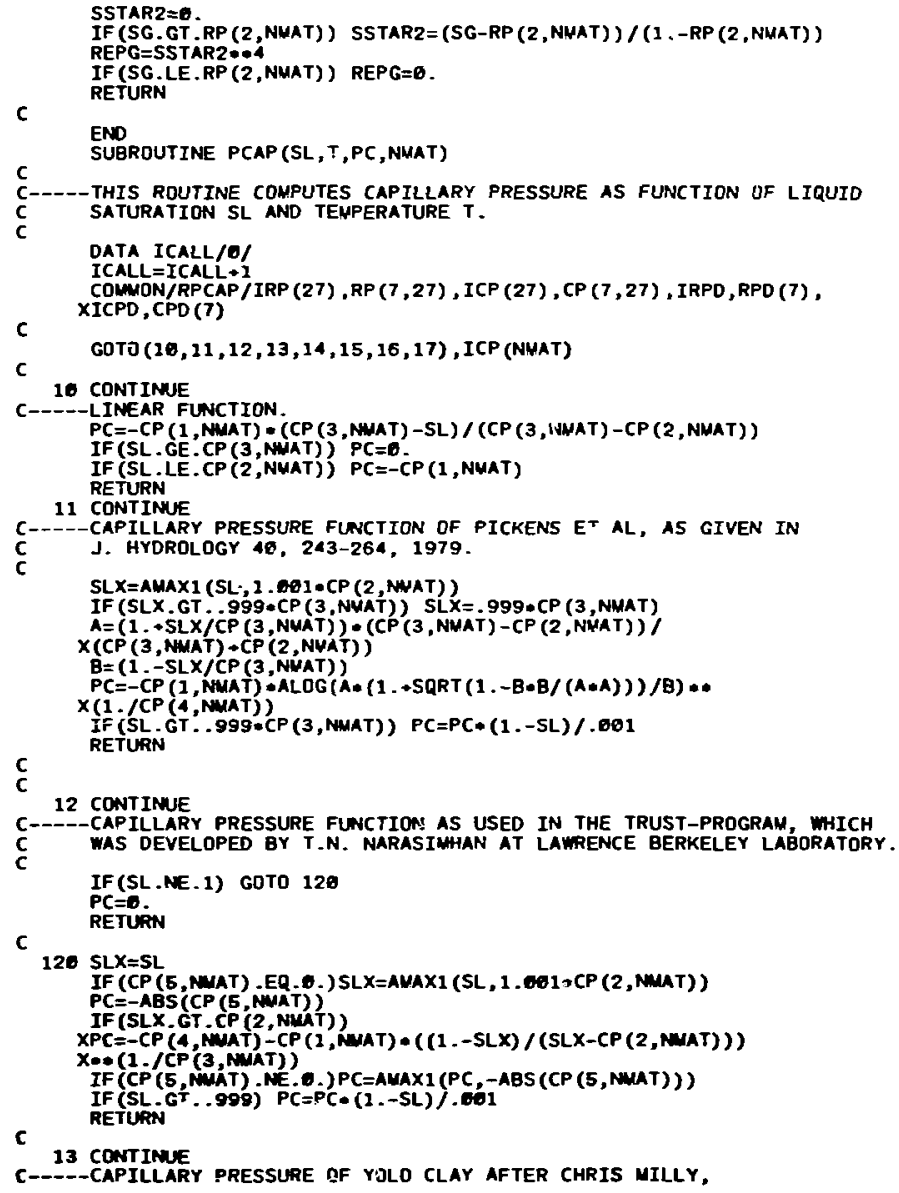




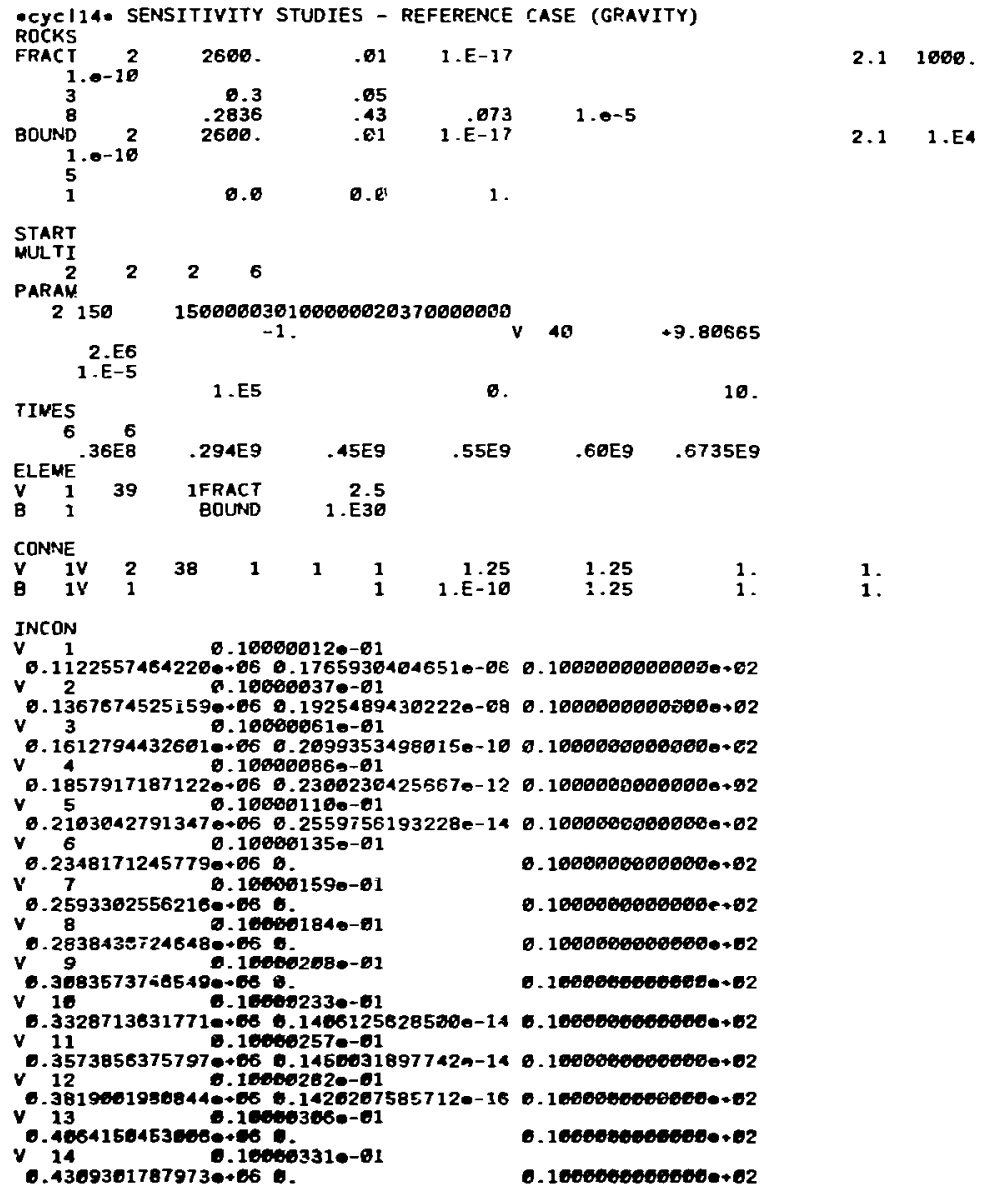

D.6 Sample TOUGH2-Input File for Reference Case. 


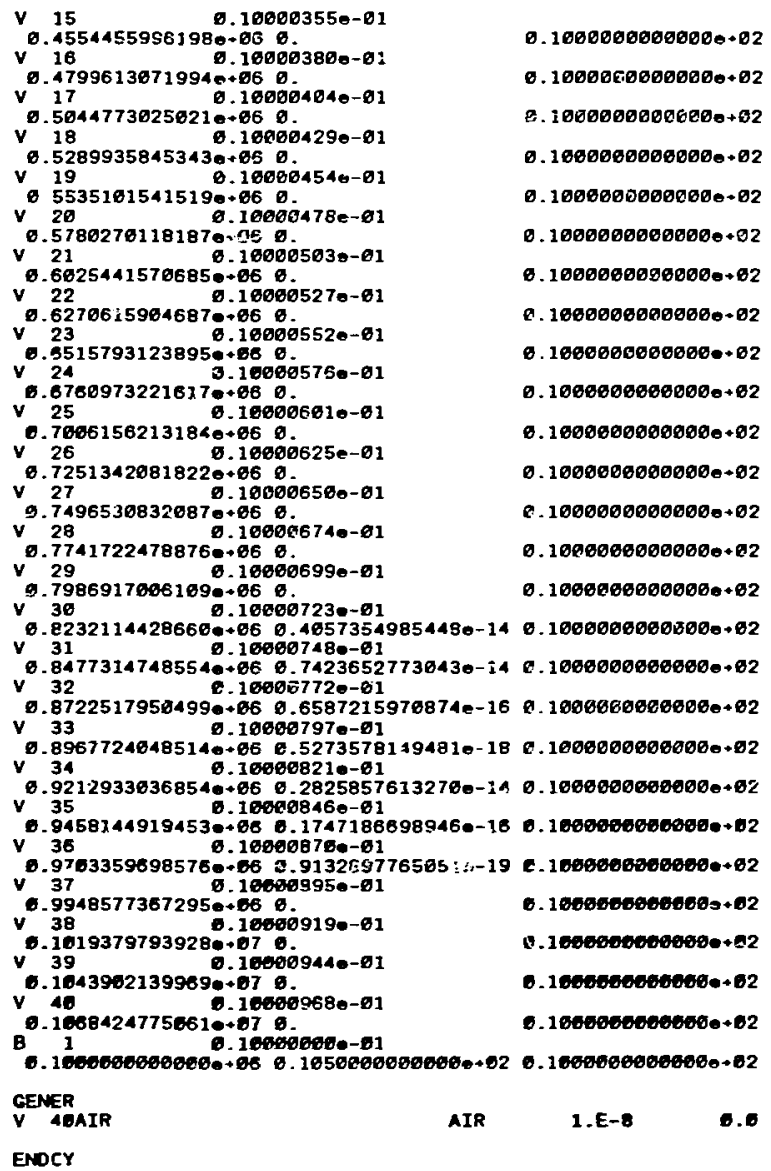

